\title{
Donor-recipient interactions drive dynamics of horizontal gene transfer via natural competence
}

Yu-Yu Cheng ${ }^{1}$, James M. Papadopoulos ${ }^{1,3}$, Tanya Falbel ${ }^{2}$, Briana M. Burton ${ }^{2}$, and Ophelia S. Venturelli ${ }^{1,2,3^{*}}$

${ }^{1}$ Department of Biochemistry, University of Wisconsin - Madison, WI, United States

${ }^{2}$ Department of Bacteriology, University of Wisconsin - Madison, WI, United States

${ }^{3}$ Department of Chemical \& Biological Engineering, University of Wisconsin-Madison

*To whom correspondence should be addressed: venturelli@wisc.edu 


\begin{abstract}
Horizontal gene transfer (HGT) in microbial communities is shaped by a complex web of abiotic and biotic interactions. We investigate the role of donor-recipient interactions on the dynamics of HGT via natural competence in a synthetic community. We show that the donor can play an active role in the gene transfer process that depends on the source of the transferred DNA. The efficiency of plasmid gene transfer depends on the abundance of the donor strain, whereas donor growth impairment augments the efficiency of chromosomal gene transfer. We show that antibiotic stress can either diminish or enhance the efficiency of gene transfer. A dynamic computational model of the system captures the impact of donor growth perturbations on gene transfer efficiency. Our results suggest that anti-HGT strategies targeting the donor strain can lead to opposing effects on the rate of HGT that depend on the microbial interaction network and source of transferred DNA.
\end{abstract}

\title{
INTRODUCTION
}

Horizontal gene transfer (HGT) is a major mechanism of genetic variation in microbial communities that enables the acquisition of new functional capabilities (1). Horizontally acquired sequences can provide a selective advantage by facilitating evolutionary adaptation to changing environments (2). Conjugation and natural transformation are prevalent processes that enable HGT in bacterial communities. Conjugation involves cell-to-cell contact between a donor and recipient cell and thus the live donor cell actively participates in the HGT process (3). By contrast, extracellular DNA (eDNA) can be acquired by a recipient cell that has activated the natural competence pathway in the absence of living donor cells (4). The capability for natural competence is widespread across Gram-positive and Gram-negative bacteria (5) and is also a common trait among bacterial pathogens (6). For example, inter-species or intra-strain gene transfer (7-9) via natural transformation is implicated in the ability of Streptococcus pneumonia (S. pneumonia) to adapt and persist on a human host. In Bacillus subtilis (B. subtilis), natural competence is a major driver of genome diversity, which has enabled this species to secure niches in animal, plant, soil and marine environments (10).

The molecular and ecological factors that determine the rate of eDNA release or transfer of DNA between donor and recipient in microbial communities are not well understood. The current paradigm for natural competence requires the presence of eDNA and recipient cells that have activated the natural competence program. However, interactions between living donor and recipient have been shown to influence HGT frequencies in microbial communities. Specific naturally competent species can exploit predation to enhance DNA release from intact or recently killed donor cells. For example, Acinetobacter baumannii, Vibrio cholerae and S. pneumoniae can play an active role in gene transfer via predation using type-VI secretion systems or bacteriocins to enhance DNA release (11-13). Further, spatial proximity was a major factor for gene transfer between specific donors and recipients using conjugation-independent mechanisms. For instance, spatial proximity was shown to enhance intra-strain gene transfer in $B$. subtilis, Porphyromonas gingivalis and Pseudomonas stutzeri, as well as inter-species gene transfer between the donor Escherichia coli (E. coli) and recipient Vibrio species (14-17). However, there are many unresolved questions about the role of donor-recipient interactions on gene transfer via natural competence in microbial communities.

The source DNA for natural transformation can be derived from the donor cell chromosome or plasmids harbored by the donor, as opposed to conjugation which is primarily selective for mobile plasmids. Plasmids or chromosome as source molecules for gene transfer exhibit differences in copy number $(18,19)$, stability in the extracellular environment $(20)$, release profiles as a function of growth stage (21-23) and molecular mechanisms of DNA uptake by the recipient cell (5). In addition to these properties, multi-copy plasmids can impose a substantial metabolic burden by sequestering intracellular cellular resources, leading to substantial changes 
in metabolic and stress response activities, cell morphology and growth rate (24-26). However, the effects of plasmid metabolic burden on rates of HGT in microbial communities has not been investigated.

To address these gaps, we investigate gene transfer dynamics in a synthetic consortium composed of the genetically manipulable donor and recipient species $E$. coli and $B$. subtilis. Using a systems-level approach, we demonstrate that donor-recipient interactions and the source of the transferred sequences (plasmid or chromosome) are major factors influencing the efficiency of HGT and its dependence on environmental factors. We find that the presence of the $E$. coli donor substantially enhances plasmid transfer compared to purified DNA, whereas the reciprocal trend is observed for chromosomal transfer. We construct dynamic computational models of species growth and HGT via natural competence to further dissect the interactions between donor and recipient in the system. To test the model predictions, we selectively modulate the growth of the donor by administering antibiotics, introducing plasmids with a range of metabolic costs or tuning the rate of cell lysis. Our data show that the abundance of the donor is positively correlated with plasmid HGT efficiency, whereas substantial donor growth impairment is required for transfer of chromosomal sequences, consistent with the model predictions. Antibiotic stress can either enhance or reduce the transfer efficiency of plasmid derived genes. In sum, our results indicate environmental perturbations that impact the growth of the donor in microbial communities can differentially impact horizontal gene transfer rates derived from plasmid or chromosomal origins.

\section{RESULTS}

\section{Temporal characterization of inter-species gene transfer in a synthetic community}

To study the factors influencing the frequency of HGT in microbial communities, we constructed a synthetic community composed of two distantly related species $E$. coli (donor) and $B$. subtilis (recipient). An integration cassette comprised of $500 \mathrm{bp}$ sequences homologous to the $B$. subtilis chromosome flanking a spectinomycin resistance gene (specR) was introduced into an $E$. coli plasmid or an erythromycin resistance gene (ermR) was integrated onto the $E$. coli chromosome. To characterize the dynamics of gene transfer, we performed time-series measurements of absolute species abundance, integration cassette extracellular DNA (eDNA) concentration and $B$. subtilis transformation frequency in the presence of purified DNA (100 ng mL-1 plasmid or chromosomal DNA) or living $E$. coli harboring the plasmid $(E c-P)$ or chromosomal cassette (Ec$C$ ) in monoculture or co-culture (Figs. 1 and 2 (A and E)). Transformation frequency or HGT efficiency was defined as the fraction of $B$. subtilis colony forming units (CFU) that were resistant to the antibiotic gene contained in the integration cassette divided by the total number of $B$. subtilis CFU. To enable control of competence activity, a second copy of the master regulator comK was introduced onto the $B$. subtilis chromosome under control of a xylose-inducible promoter (27).

$B$. subtilis exhibited a lower growth rate and carrying capacity in the presence of $E$. coli, whereas the presence of $B$. subtilis did not impact the growth of $E$. coli, indicating a negative interspecies interaction from $E$. coli to $B$. subtilis (Fig. 2, B and F). Although the $E$. coli growth response was not altered in the presence of $B$. subtilis, the eDNA concentration was substantially higher in the co-culture than in the E. coli monoculture (Fig. 2, C and G). Notably, the efficiency of plasmid transfer was significantly enhanced across all time points by up to $\sim 480$-fold at $8 \mathrm{hr}$ in the co-culture compared to $B$. subtilis monoculture supplemented with purified plasmid DNA, demonstrating that inter-species interactions were a critical determinant of HGT efficiency (Fig. 2D). Corroborating this result, the HGT efficiency of a co-culture containing native comK $B$. subtilis PY79 (WT B. subtilis) and Ec-P was substantially higher than the WT $B$. subtilis monoculture exposed to purified plasmid DNA, indicating that the observed increase in HGT efficiency was not attributed to synthetic control of the competence pathway (fig. S1A). In addition, E. coli negatively impacted WT $B$. subtilis growth by a smaller magnitude compared to engineered $B$. subtilis, suggesting that the xylose-induced competence-dependent growth arrest may further reduce the 
competitive ability of $B$. subtilis in the community (Fig. 2 (B and F) and fig. S1B). By contrast, transformants were not detected at any time point in the $E c-C$ co-culture and a low transformation frequency was observed in the $B$. subtilis monoculture in the presence of purified chromosomal DNA, indicating that the efficiency of chromosomal transfer was reduced in the presence of the donor species (Fig. 2H).

The critical role of the $E$. coli donor in enhancing plasmid transfer efficiency suggested that plasmid transfer may involve mechanisms akin to conjugation. Since natural competence involves eDNA released from the donor, we tested whether eDNA was required for inter-species gene transfer by introducing a DNA degrading enzyme (DNase I) into the co-culture. In the presence of DNase I, no transformants were detected in the Ec- $P$ and $B$. subtilis co-culture, corroborating that HGT occurred via natural transformation (fig. S2A).

Since spatial proximity between donor and recipient was shown to enhance gene transfer by natural competence in other systems $(11,12,14,17)$, we next sought to characterize the effect of spatial proximity in the Ec-P and $B$. subtilis co-culture. Ec-P and $B$. subtilis were physically separated by a $0.4 \mu \mathrm{m}$ filter, which prevented cell-to-cell contact while allowing exchange of diffusible molecules (fig. S2B). The HGT efficiency of the physically separated co-culture condition was 25-fold lower compared to the well-mixed condition in the absence of the filter, indicating that spatial proximity between donor and recipient enhanced HGT efficiency.

We next used fluorescence microscopy to visualize the spatial distribution of $E$. coli and $B$. subtilis cells in the mixed culture. The RFP-labeled $E$. coli and GFP-labeled $B$. subtilis were in close spatial proximity after $3 \mathrm{hr}$ (fig. S2C). To quantify the spatial distribution of $B$. subtilis in the presence and absence of $E$. coli, each image was partitioned into regions of interest (ROI) containing single or groups of cells in close spatial proximity. Our results showed that $99 \%$ of $B$. subtilis (at least 1000 cells) were found in close proximity to $E$. coli and the number of cells within $\mathrm{ROI}$ containing both species was higher on average compared to the number of cells within ROI containing a single species (fig. S2D). In addition, the average number of $E$. coli cells within $E$. coli only ROI was higher than the number of $B$. subtilis in $B$. subtilis only ROI (fig. S2D). These data suggest that $E$. coli can self-aggregate in the presence or absence of $B$. subtilis, whereas aggregation of $B$. subtilis occurs more frequently in the presence of $E$. coli. In sum, these data suggest that spatial proximity and cell-to-cell contact between donor and recipient may influence plasmid transfer efficiency via natural competence.

The $B$. subtilis competence developmental program is regulated by a complex and dynamic regulatory network that activates the pathway in a subpopulation of cells in response to specific extracellular signals (28). Therefore, we sought to test whether the presence of $E$. coli impacted the activation response of the competence pathway. To quantify the expression of the competence pathway, we performed time-series flow cytometry measurements of $B$. subtilis harboring a comK promoter $\left(P_{\text {comk }}\right)$ fused to GFP in the presence and absence of $E c-P$ labeled with constitutive RFP. In the presence and absence of $E c-P$, the fraction of cells expressing GFP (GFP-ON state) increased and then decreased over time, consistent the previously characterized transient activation of the pathway in a sub-population of cells (fig. S3) (28). These data suggest that the observed enhancement of plasmid transfer efficiency in the presence of $E c-P$ was not attributed to changes in the activation response of competence pathway across the population.

We next explored whether the differences in HGT efficiencies in the co-culture compared to monoculture conditions could be explained by variations in eDNA concentration. The eDNA concentrations observed in the co-cultures and monocultures (100 $\mathrm{ng} \mathrm{mL}^{-1}$ purified DNA) were in the saturated regime of the eDNA-transformation frequency dose-response curve $\left(\sim 10^{2}-10^{3} \mathrm{ng}\right.$ $\mathrm{mL}^{-1}$ for plasmid and $\sim 10^{0}-10^{2} \mathrm{ng} \mathrm{mL} \mathrm{L}^{-1}$ for chromosome) (horizontal lines in Fig. 2I), suggesting that eDNA concentration was not a limiting factor for gene transfer. After accounting for differences in gene copy numbers, the chromosome and plasmid transformation frequency were similar for the $B$. subtilis monoculture exposed to purified DNA, with a moderate and statistically 
significant increase in efficiency for chromosome than plasmid derived genes (Fig. 2J). These data suggest the majority of the differences in the efficiency of chromosomal and plasmid gene transfer in the $B$. subtilis monoculture condition could be explained variation in gene copy number, as opposed to other properties of the DNA including topology (e.g. circular, linear or strandedness), size, stability, or sequence. By ruling out $B$. subtilis competence activity, eDNA concentration and properties as major determinants of transformation frequency in the synthetic community, we next investigated how the inter-species interaction between $E$. coli and $B$. subtilis influenced HGT dynamics.

\section{Dynamic computational modeling of community dynamics and HGT}

To interrogate the mechanisms that influence gene transfer, we developed a dynamic computational model that represents species growth modified by inter-species interactions, eDNA release and HGT (Supplementary Materials). The model captures the temporal changes in live E. coli $(E)$, dead $E$. coli $(E d)$, B. subtilis $(B)$, eDNA $(G)$, and transformed $B$. subtilis (Bt) (Fig. 3A). We represent species growth dynamics and microbial interactions using terms from the LotkaVolterra model (29). To account for the time-delays in eDNA release relative to $E$. coli growth (Fig. 2, $\mathbf{B}, \mathbf{C}, \mathbf{F}$, and $\mathbf{G}), E$ is converted into $E d$ at a constant rate and $E d$ releases $G$ as a function of $B$ and $E d$. Our data showed that the extracellular concentration of plasmid increased in the $B$. subtilis monoculture condition, whereas the extracellular chromosome concentration displayed the reciprocal temporal trend (Fig. 2, C and G). To capture these temporal trends, we assume that $G$ can be produced at a rate proportional to $B$ and degraded at a rate proportional to $B$ or $E$. In addition, we assume the transformation frequency of $B$ depends on $E$ and the presence of $G$, to represent the observed differences in transformation frequency in co-culture and monoculture conditions (Fig. 2, D and H).

The model was fit to the time-series measurements of CFU, eDNA, and transformation frequency and was able to recapitulate the temporal changes across conditions (Fig. 2, B, C, D, $\mathbf{F}, \mathbf{G}$, and $\mathbf{H}$ ). To further understand the differences in molecular mechanisms between the $E c-P$ and $E c-C$ co-cultures, we computed the fold-change of each inferred parameter between the two models (Fig. 3A). The inter-species interaction coefficient $\alpha_{e b}$ representing the impact of $B$ on the growth rate of $E$ was substantially larger in magnitude in the $E c-P$ model compared to the $E c-C$ model, suggesting that plasmid metabolic burden can alter the magnitude of inter-species competition by reducing the fitness of $E$. coli, which in turn enhances the competitive ability of $B$. subtilis. In addition, the chromosome degradation rate $\gamma_{g}$ was significantly larger than plasmid, consistent with the enhanced persistence of plasmid than chromosomal DNA over time in the environment (20). To investigate the contribution of each parameter to the efficiency of HGT, each parameter was perturbed by $\pm 20 \%$ and the transformation frequency, defined as the ratio of $B t$ to the sum of $B$ and $B t$, was determined at $6 \mathrm{hr}$ (Fig. 3B). Notably, increasing the death rate of $E c-P$ or $E c-C\left(\gamma_{e}\right)$ has opposing effects on the HGT efficiency in the model.

To test the model predicted effects of $E$. coli death rate on HGT efficiency, we introduced an IPTG-inducible phage $\varphi$ X174 lysis gene $E$ into $E c-P$ and $E c-C$ to program the donor lysis rate (Fig. 3, C and E). The eDNA concentration increased substantially as a function of IPTG, demonstrating that enhanced expression of lysis gene $E$ triggered donor lysis and eDNA release (fig. S4). In both communities, the abundance of $B$. subtilis and $E$. coli displayed opposing trends as a function of IPTG, consistent with relationship between $\gamma_{e}$ and species abundance in the models (Fig. 3, C, D, E and F). Notably, the transformation frequency in the $B$. subtilis and Ec-P co-culture exhibited a decreasing trend with IPTG concentration, consistent with the relationship between $\gamma_{e}$ and transformation frequency in the plasmid model (Fig. 3, C and D). The reciprocal pattern was observed for the $E c-C$ community wherein transformants were only detected at high IPTG concentrations, reflecting a moderate increase in transformation frequency as a function of $\gamma_{e}$ in the chromosome model (Fig. 3, E and F). These data demonstrated that substantial growth 
inhibition of $E c-C$ was required to observe transformants above the detection limit. Inhibition of $E c-C$ growth in the presence of high IPTG concentrations enhanced both eDNA concentration and $B$. subtilis abundance, suggesting that both of these factors were critical for efficient chromosomal transfer (Fig. 3 (E and F) and fig. S4B).

To further test the predictions of the models, we varied both the initial proportion and total density of $B$. subtilis and $E c-P$ or $E c-C$ in the co-cultures and measured transformation frequency at $6 \mathrm{hr}$. Our model captured the trends across the different conditions for the Ec-P co-culture, including an enhanced number of $B$. subtilis transformants in conditions with high initial species densities (fig. S5A). The measured transformation frequency for the $E c-C$ co-culture was below the level of detection across the majority of conditions, reflecting the model prediction of a low number of $B$. subtilis transformants across all conditions (fig. S5B). In sum, our model accurately forecasted the differential roles of donor cell lysis rates in the $E c-P$ and $E c-C$ co-cultures, as well as the effects of initial species densities on HGT efficiency.

The eDNA release rates $\left(\gamma_{e}\right.$ and $\left.\lambda_{d 1}\right)$ are larger for $E c-P$ than $E c-C$, suggesting that $E$. coli lysis is enhanced in the presence of the plasmid (Fig. 3A and table S1). To test this prediction, we varied the degree of plasmid-mediated metabolic burden by introducing a set of plasmids with different copy numbers and sizes into $E c-C$ (Fig. 3G). Corroborating the plasmid metabolic burden effect, the $E$. coli doubling time was correlated with the predicted number of plasmid DNA base pairs per cell (fig. S6). The extracellular chromosome concentration increased with plasmid

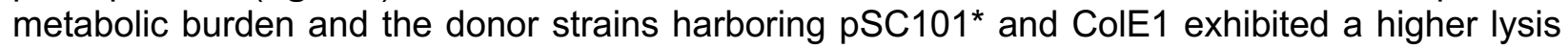
rate in the presence of $B$. subtilis compared to monoculture (Fig. 3G). Notably, transformants were not observed in the presence of any of the $E c-C$ donor strains except the strain harboring the plasmid with the highest metabolic cost (pBB275), demonstrating that plasmid metabolic burden could substantially enhance the efficiency of chromosomal HGT in microbial communities (Fig. $3 \mathrm{H})$. In sum, the abundance of the recipient, lysis rate of the donor and plasmid metabolic burden enhanced the efficiency of chromosomal transfer in the synthetic microbial community.

\section{Impact of antibiotics that selectively target donor growth on horizontal gene transfer}

Antibiotics have been shown to impact the frequencies of HGT via induction of conjugation and competence pathways (30-32). To investigate how antibiotics impact gene transfer dynamics in the synthetic community, we quantified the temporal change in eDNA release, absolute species abundance and HGT efficiency in the co-culture in response to the antibiotics streptomycin (bactericidal) or chloramphenicol (bacteriostatic). We used sub-lethal antibiotic concentrations for $B$. subtilis to study how selective inhibition of the donor growth rate impacts HGT efficiency (fig. S7). In response to streptomycin, the growth of $E c-P$ and $E c-C$ were impaired, whereas the growth of $B$. subtilis was enhanced compared to the no antibiotic control, consistent with an attenuation of the inhibitory interaction impacting $B$. subtilis (Figs. 3A, 4 (A, B, E, and F), and table S1). The eDNA concentration was higher in the $E c-P$ and $E c-C$ co-cultures in the presence of streptomycin, indicating that streptomycin enhanced the lysis rate of $E$. coli (Fig. 4, C and G). Although the concentration of eDNA was augmented in the presence of streptomycin, the transformation frequency in the Ec-P co-culture was significantly reduced compared to the no antibiotic control (Fig. 4D), corroborating the key role of Ec-P abundance on HGT efficiency (Fig. 3, C and D). By contrast, the transformation frequency in the $E c-C$ co-culture in the presence of streptomycin was enhanced compared to the no antibiotic condition, consistent the major contribution of the abundance of the recipient to chromosomal transfer (Figs. $3 \mathbf{E}$ and $\mathbf{4 H}$ ). In the presence of streptomycin, $E c-P$ failed to grow in the community, whereas $E c-C$ persisted over time, indicating that plasmid metabolic burden not only reduced $E$. coli growth and enhanced lysis, but also magnified antibiotic sensitivity (Figs. 4, A and E).

Exposure to sub-lethal concentration of chloramphenicol had opposing effects on the plasmid and chromosomal temporal release profiles, while consistently reducing the growth rate of E. coli (Fig. 4, A, C, E, and G). These temporal trends are consistent with the differential impact 
of sub-lethal chloramphenicol concentrations on the stringent response, which leads to enhanced replication of plasmids regulated by relaxed control while inhibiting chromosomal replication (33). The presence of chloramphenicol had differential impacts on HGT efficiency in the $E c-C$ or $E c-P$ co-cultures by enhancing or not altering gene transfer, respectively (Fig. 4, D and H). In sum, our results showed that both the cold-shock antibiotic streptomycin or heat-shock antibiotic chloramphenicol consistently enhanced chromosome transfer even though the rate of eDNA release exhibited opposing temporal responses (higher and lower donor lysis rate in the presence of streptomycin or chloramphenicol, respectively than the no antibiotic condition) (Fig. 4H) (34). However, streptomycin and chloramphenicol exhibited differential effects on plasmid gene transfer by either reducing or not altering HGT efficiency (Fig. 4D). Therefore, the mechanism of action of the antibiotic combined with the origin of the transferred DNA molecule dictated the role of the antibiotic on inter-species gene transfer in the synthetic community.

\section{Investigating key molecular and ecological factors shaping gene transfer}

We next sought to understand the relationships between different measured variables of the system across all experiments to provide insight into the factors shaping inter-species plasmid or chromosome gene transfer. The abundance of $E c-C$ and $B$. subtilis were negatively correlated, consistent with the inferred negative inter-species interaction $\alpha_{b e}$ of $E$ on the growth rate of $B$ in the $E c-C$ model (Fig. 3A, fig. S8A, and table S1). The plasmid transfer frequency was positively correlated with $E$. coli abundance (Pearson correlation coefficient $r=0.78, p$-value $=9.37 \mathrm{e}-7$ ), consistent with our model's prediction (Fig. 5 (A and B)). By contrast, the $E c-C$ abundance was not correlated to transformation frequency, mirroring the model prediction (Fig. 5, C and D). Indeed, the efficiency of plasmid transfer was maximized for high $E$. coli abundance, whereas chromosome transfer displayed the opposite relationship with $E$. coli abundance and required strong inhibition of E. coli growth to observe transformants (Fig. 5, E and G). The Pearson correlations between plasmid or chromosome eDNA concentration and transformation frequency were not statistically significant, suggesting that eDNA was not limiting for HGT in these conditions (fig. S8, B and C).

Inhibition of $E$. coli growth by antibiotic stress and programmed cell lysis resulted in an increase in $B$. subtilis abundance by weakening the strong negative interaction impacting $B$. subtilis (Figs. 3 (C and $E$ ) and 4 (B and F)). The abundance of $E c-P$ or $E c-C$ and the product of $B$. subtilis abundance and time-lagged eDNA concentration were negatively correlated (Pearson correlation coefficient $r=-0.61, p$-value $=0.00163$ for plasmid and $r=-0.89, p$-value $=2.94 \mathrm{e}-11$ for chromosome) (Fig. 5, E and G), reflecting the trend in the models (Fig. 5, F and H). The strongest negative correlation between $E$. coli abundance and chromosome eDNA concentration occurred for eDNA concentration measured four hours earlier, suggesting a time delay between cell death and eDNA release (fig. S8D). In sum, our results demonstrate that the abundance of the $E$. coli donor was a key determinant of efficient plasmid transfer. However, our data suggests efficient chromosome transfer required both sufficient eDNA concentration and $B$. subtilis abundance in the community (Fig. 5G). Therefore, our results suggest the strong negative interaction from $E c-C$ to $B$. subtilis is the major factor that impeded chromosomal HGT in the synthetic community and thus environmental perturbations that alleviated microbial competition and increased donor cell lysis rates substantially enhanced chromosomal transfer.

\section{DISCUSSION}

The intracellular network and environmental signals that control the expression of the natural competence pathway in $B$. subtilis in monoculture conditions have been extensively characterized $(35,36)$. The current paradigm for gene transfer via natural competence in $B$. subtilis is that the recipient drives control of this process by activating competence gene expression in a fraction of the population in response to specific environmental signals_(37). However, B. subtilis has evolved 
in diverse microbial communities shaped by a complex and dynamic web of abiotic and biotic interactions that impact the rates of HGT_(10). Reduced complexity consortia composed of genetically manipulable species allow precise control of environmental, ecological and intracellular network factors and are thus powerful systems to dissect the variables shaping the rate of HGT.

Using a detailed and quantitative analysis of a synthetic microbial community, our results challenge the current paradigm of recipient driven control of gene transfer via natural competence by demonstrating that the donor strain $E$. coli was a major driver of plasmid transfer as opposed to the concentration of eDNA (14). Notably, efficient chromosomal HGT required strong growth inhibition of the donor, which in turn increased the abundance of $B$. subtilis by impairing the strength of microbial competition and eDNA concentration. Therefore, our results demonstrate that the source of the transferred DNA determined the effect of the donor strain on the rates of HGT in the community. The emergence of antibiotic resistance pathogens via HGT has accelerated in recent years and is an imminent threat to global public health (6). Selective targeting of donor strains harboring antibiotic resistance genes has been proposed as a strategy to reduce the propagation of these genes through microbial communities $(38,39)$. However, our results indicate that altering the abundance and lysis rates of donor strains could either potentiate or reduce the rate of HGT via natural competence depending on whether the antibiotic resistance genes are derived from plasmid or chromosome origin.

Specific antibiotics have been shown to induce competence gene expression or enhance transformation frequencies in $S$. pneumoniae, Legionella pneumophila and $B$. subtilis monocultures due to activation of stress responses or variation in gene copy numbers $(14,31,32$, 40). However, the effect of an antibiotic on the efficiency of gene transfer depends on both the specific mechanism of action and the effective concentration. Using the synthetic community, we found that the bactericidal antibiotic streptomycin substantially reduced inter-species plasmid transfer, whereas the bacteriostatic antibiotic chloramphenicol did not impact the efficiency of this process. By contrast, both antibiotics enhanced inter-species transfer of the chromosomal derived genes. Therefore, in a microbial community context, antibiotic stress could either diminish or enhance inter-species gene transfer, which has implications for the design of antibiotic treatments. Taken together, these results demonstrate that the plasmid or chromosome origin of transferred DNA sequences is a major determinant of the impact of antibiotic stress on gene transfer due to potential differences in the molecular mechanisms of HGT. Based on these results, the use of selective chemical inhibitors that target natural competence activities in the recipient and growth of the donor strain could be a promising strategy to reduce the rate of horizontal gene transfer of plasmid derived genes in microbial communities_(41). By contrast, the rate of HGT of chromosomal derived genes could be reduced by exploiting microbial competition to exclude the recipient while promoting the growth of the donor.

Our results demonstrated that spatial proximity of $E$. coli and $B$. subtilis substantially enhanced plasmid transfer. Consistent with this result, cell-to-cell contact and spatial proximity have been shown to play an important role in inter-species and intra-strain gene transfer via natural competence in several other microbial communities $(11,12,14,17)$. The molecular mechanisms driving these physical associations are not understood, with the exception of gene transfer mediated by the contact-dependent type VI secretion system (T6SS). E. coli has been shown to self-aggregate and adhere to different species including yeast and mammalian cells (42-44), suggesting that this property could influence HGT efficiency in the synthetic community. Future work could investigate the molecular mechanisms and genetic determinants of cell-to-cell contact in the HGT process via natural competence in microbial communities.

We showed that plasmid metabolic burden reduced the growth rate of the donor and enhanced cell lysis, leading to an increase in the rate of eDNA release and chromosomal HGT. Future work will investigate if plasmid metabolic burden leads to higher lysis and gene transfer efficiencies across diverse bacterial species. If this phenomenon is generalizable to other species, 
the frequency of transferring genetic information encoded on the chromosome to other species via natural competence may be higher for strains that harbor metabolically costly plasmids. Therefore, prediction of potential hub donor species in HGT networks could leverage metagenomics linking plasmids to host genomes (45). In addition, the effect of plasmid metabolic burden on cell lysis presents potential challenges for biocontainment of engineered DNA sequences in the environment (46).

The reduced complexity of the synthetic community enabled a detailed and quantitative investigation of molecular and ecological factors influencing gene transfer dynamics. However, this community does not capture the taxonomic diversity of natural microbiomes. In natural communities, the donor and/or recipient taxa are largely unknown as well as the effects of environmental perturbations such as antibiotic stress on HGT events. Horizontal gene transfer events can be identified in natural communities in situ via time-series analysis of strain-level genome sequences using high-throughput chromosomal confirmation capture (Hi-C) (47). Future work could couple such multiplexed measurements of HGT within microbiomes with network inference techniques to decipher the complex web of interactions between abiotic and biotic factors that drive HGT. A deeper and quantitative understanding of the ecological and molecular factors shaping HGT efficiency could be exploited to design anti-HGT microbiome interventions to reduce the propagation of antibiotic resistance and virulence genes in microbiomes.

\section{MATERIALS AND METHODS}

Strain and plasmid construction

The strain background for $E c-P$ and $E c-C$ was $E$. coli MG1655. For flow cytometry analysis, RFP driven by the $P_{\mathrm{J} 23100}$ constitutive promoter was introduced onto the $E c-P$ genome downstream of caiE gene using CRISPR gene editing techniques (48). Ec-P was transformed with the plasmid pBB275 which contains a spectinomycin resistance gene (specR) flanked by two 500 bp ycgO sequences homologous to the $B$. subtilis PY79 genome. To construct $E c-C$, an erythromycin resistance gene (ermR) and GFP driven by the $P_{\text {veg }}$ constitutive promoter flanked by two 500 bp $y v b J$ sequences homologous to $B$. subtilis PY79 were integrated onto the $E$. coli genome (downstream of caiE gene). For programmable E. coli lysis, the plasmid pYC01 harboring the $\varphi X 174$ lysis gene $E$ driven by an IPTG-inducible promoter was used to control the donor lysis rate. The plasmid pYC01 was constructed from pCSaE500 (Addgene \#53182) by replacing the lysis gene promoter with $\mathrm{P}_{\mathrm{A} 1 \mathrm{lacO}-1}$ promoter (49). To characterize plasmid metabolic burden, ColE1 (pBbE2k-RFP), p15A (pBbA2a-RFP), and pSC101* (pBbS2c-RFP) were derived from the BglBrick library were individually transformed into $E c-C$ using standard chemical transformation techniques (50). To increase the transformation efficiency of $B$. subtilis PY79 in LB medium, a xylose-inducible comK was integrated into amyE locus (27). To monitor the competence activity,

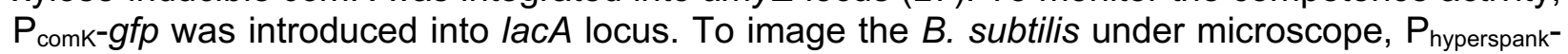
gfp (51) was integrated into lacA locus. All DNA oligos used for cloning were synthesized by Integrated DNA Technologies (IDT). The list of plasmids and strains used in this study can be found in table S3. The estimated copy numbers per cell for pSC101* ${ }^{*}$ p15A, ColE1 and pBB275 were 3-4, 20-30, 50-70 and 50-70, respectively (49). The plasmid pBB275 was $\sim 1.5 \mathrm{~kb}$ larger in size than ColE1 and shared the same origin of replication. To normalize for differences in DNA copy numbers, the eDNA copy number was calculated by NEBioCalculator with pBB275 size $4927 \mathrm{bp}$ and $E$. coli MG1655 genome size $4639675 \mathrm{bp}$.

\section{Bacterial culturing experiments}

For bacterial culturing experiments, cells were first inoculated from a $-80^{\circ} \mathrm{C}$ glycerol stock into 4 $\mathrm{mL}$ Lennox LB media (Sigma-Aldrich) containing antibiotics for selection and cultured at $37^{\circ} \mathrm{C}$ for 12 hours. The OD600 was measured by NanoDrop One (Thermo Fisher Scientific) and the cells were diluted to an OD600 of 0.1 in $10 \mathrm{~mL}$ LB supplemented with $50 \mathrm{mM}$ xylose (Thermo Fisher 
Scientific) in $14 \mathrm{~mL}$ Falcon ${ }^{\mathrm{TM}}$ Round-Bottom Tubes (Thermo Fisher Scientific) and cultured at $37^{\circ} \mathrm{C}$ with shaking. For the $B$. subtilis monoculture experiments, the media was supplemented with $100 \mathrm{ng} \mathrm{mL}^{-1}$ purified E. coli plasmid or genomic DNA. Plasmid and genomic DNA were purified using a Plasmid Miniprep Kit (Qiagen) and DNeasy Blood \& Tissue Kit (Qiagen). Samples were collected every two hours for measuring colony forming units (CFU), extracellular DNA, and flow cytometry. CFU measurements were performed by diluting the cell culture into phosphatebuffered saline (PBS) buffer (Sigma-Aldrich) and plating onto LB agar plates containing $100 \mu \mathrm{g}$ $\mathrm{ml}^{-1}$ spectinomycin (GoldBio) for Ec-P or $1 \mu \mathrm{g} \mathrm{mL}-1$ erythromycin (Sigma-Aldrich) and $25 \mu \mathrm{g} / \mathrm{mL}$ lincomycin (VWR) for Ec-C. In the Ec-P co-culture, $B$. subtilis were selected for on LB agar plates containing $5 \mu \mathrm{g} \mathrm{mL}^{-1}$ chloramphenicol (Sigma-Aldrich), $1 \mu \mathrm{g} \mathrm{mL}^{-1}$ erythromycin, and $25 \mu \mathrm{gL}^{-1}$ lincomycin. In the $E c-C$ co-culture, $B$. subtilis were selected for on LB agar plates containing $5 \mu \mathrm{g}$ $\mathrm{mL}^{-1}$ chloramphenicol and $10 \mu \mathrm{g} \mathrm{mL}^{-1}$ kanamycin (Sigma-Aldrich). B. subtilis transformed with plasmid pBB275 was selected on LB agar plates containing $5 \mu \mathrm{g} \mathrm{mL}^{-1}$ chloramphenicol, $1 \mu \mathrm{g} \mathrm{mL}-$ ${ }^{1}$ erythromycin, $25 \mu \mathrm{g} \mathrm{mL}^{-1}$ lincomycin, and $100 \mu \mathrm{g} \mathrm{mL}^{-1}$ spectinomycin. $B$. subtilis transformed with $E c-C$ chromosomal DNA was selected on LB agar plates containing $5 \mu \mathrm{g} \mathrm{mL} \mathrm{L}^{-1}$ chloramphenicol, $10 \mu \mathrm{g} / \mathrm{mL}$ kanamycin, $1 \mu \mathrm{g} \mathrm{mL}^{-1}$ erythromycin, and $25 \mu \mathrm{g} \mathrm{mL}^{-1}$ lincomycin. Agar plates were incubated at $37^{\circ} \mathrm{C}$ overnight and colonies were counted after 24 hours. Transformation frequency was defined as the ratio of the number of $B$. subtilis CFU per $\mathrm{mL}$ to the total number of $B$. subtilis CFU per $\mathrm{mL}$. The detection limit of transformants was $1 \mathrm{CFU} \mathrm{mL}^{-1}$ transformed $B$. subtilis divided by the total $B$. subtilis CFU $\mathrm{mL}^{-1}$.

To test if the horizontal gene transfer occurred via natural transformation, 2 units $\mathrm{mL}^{-1}$ DNase I (Thermo Fisher Scientific) was added to the culture. According to the manufacturer, the quantity of 1 unit of DNase I can degrades $1 \mu \mathrm{g}$ of plasmid DNA in $10 \mathrm{~min}$ at $37^{\circ} \mathrm{C}$. Therefore, we assumed that 2 units $\mathrm{mL}^{-1}$ DNase I can fully degrade $100 \mathrm{ng} \mathrm{mL}^{-1}$ plasmid administered to the $B$. subtilis monoculture and extracellular plasmid released in co-culture the with $E c-P$.

\section{Quantitative real-time PCR measurements of extracellular DNA}

To quantify eDNA in the cell culture, an aliquot of the cell culture was centrifuged at $5000 \times \mathrm{xg}$ for 5 min and the supernatant was filtered using a $0.2 \mu \mathrm{m}$ Whatman Puradisc Polyethersulfone Syringe Filter (GE Healthcare). To measure eDNA concentration by qPCR, the filtered supernatant was added to a PCR reaction containing the SsoAdvanced ${ }^{T M}$ Universal Probes Supermix (Bio-Rad) and TaqMan primers with probes 5'FAM/ZEN/3'IABkFQ (Integrated DNA Technologies). The TaqMan primers were designed to amplify a $129 \mathrm{bp}$ region of the $s p e c R$ gene on the plasmid pBB275 in Ec- $P$, and a 145bp region of the ermR on the $E c-C$ genome. The plasmid and genomic DNA standards were prepared as follows: plasmid was purified from $E$. coli $\mathrm{DH} 5 \alpha$ using the Plasmid Miniprep Kit (Qiagen) and genomic DNA was extracted from Ec-C using DNeasy Blood \& Tissue Kit (Qiagen). The extracted DNA was quantified by Quant-iT dsDNA Assay Kit (Thermo Fisher Scientific) and then serially diluted to span a broad range of concentrations. The qPCR reactions were run on the CFX Connect Real-Time PCR Detection System (Bio-Rad). The eDNA concentrations were determined by interpolating the standard curve mapping DNA concentration to Cq values.

\section{Flow cytometer measurements of $B$. subtilis competence activity}

To characterize competence activity in $B$. subtilis, a modified $B$. subtilis PY79 strain harboring

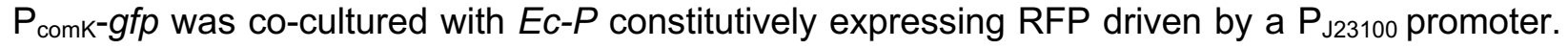
Fluorescence was measured on a LSRFortessa X-20 Flow Cytometer (BD Biosciences). A blue $(488 \mathrm{~nm})$ laser was used to quantify GFP and emission was detected using a 530/30 nm filter and a yellow-green (561 nm) laser was used to excite RFP and emission was detected using a 610/20 $\mathrm{nm}$ filter. The GFP and RFP fluorescence distribution was displayed using the FlowJo software. The RFP-OFF and RFP-ON cells were separated at $0 \mathrm{hr}$, but they merged after 2 hours (fig. S3B). 
To quantify the fraction of GFP-ON cells, a threshold in GFP (5000 au) was selected to separate the OFF and ON states of the bimodal fluorescence distribution of the $B$. subtilis monoculture in the presence of $50 \mathrm{mM}$ xylose and $100 \mathrm{ng} \mathrm{mL}^{-1}$ purified plasmid DNA (fig. S3A) (52). However, the choice of the threshold does not affect the qualitative trend between monoculture and co-culture conditions. Data analysis was performed using custom MATLAB scripts.

Plate reader measurement of bacterial growth with antibiotics

To determine the sub-lethal concentration for $B$. subtilis and effect on $E$. coli growth, the overnight culture of $E c-P$ and $B$. subtilis (with chloramphenicol resistance gene) was diluted to OD600 equal to 0.1 in LB media with different concentrations of streptomycin (Sigma-Aldrich) or chloramphenicol (Sigma-Aldrich). The cell cultures were incubated in a 96-well plate (VWR, Catalog Number 82050-744) with shaking and sealed with the Breathe-Easy Adhesive Microplate Seals (Thermo Fisher Scientific) in a TECAN Spark 10M Multimode Microplate Reader and OD600 measurements were performed every five minutes.

\section{Transwell experiment}

To test if spatial proximity between donor and recipient enhances plasmid-mediated gene transfer, cells were grown in 6-well Nunc Cell Culture Plate (Thermo Fisher Scientific). Ec- $P$ and B. subtilis or $100 \mathrm{ng} \mathrm{mL}^{-1}$ purified plasmid DNA (pBB275) and $B$. subtilis were separated by a $0.4 \mu \mathrm{m}$ filter. The plate was sealed with Breathe-Easy Adhesive Microplate Seals (Thermo Fisher Scientific) and shaken incubated at $37^{\circ} \mathrm{C}$ with shaking. After $6 \mathrm{hr}$, samples from the two compartments separated by the filter were plated on LB agar plates containing the appropriate antibiotics and colonies were counted after $24 \mathrm{hr}$ incubation at $37^{\circ} \mathrm{C}$. Our controls showed that $0.01 \%$ of $E$. coli were observed in the $B$. subtilis compartment at $6 \mathrm{hr}$, demonstrating that the filter functioned as a physical barrier to cell diffusion between compartments.

\section{Fluorescent microscopy imaging of bacteria}

To image fluorescently labeled $E$. coli and $B$. subtilis in the co-culture, $4 \mu \mathrm{L}$ of the cell cultures were transferred to the glass slide after $3 \mathrm{hr}$ incubation with shaking at $3 \mathrm{hr}$ at $37^{\circ} \mathrm{C}$. To immobilize the cells for imaging, $5 \mu \mathrm{L}$ of $0.1 \%(\mathrm{w} / \mathrm{v})$ Poly-L-lysine (Millipore Sigma) was spread evenly on the glass slide. Pipetting of the cultures was minimized to prevent the disruption of the physically associated cells. Single cells were imaged by Nikon Eclipse Ti Microscope with 40X magnification. Fluorescence was imaged using the following filters (Chroma): GFP: $470 \mathrm{~nm} / 40 \mathrm{~nm}$ (ex), $525 / 50 \mathrm{~nm}$ (em) or RFP: $560 \mathrm{~nm} / 40 \mathrm{~nm}$ (ex), 630/70 nm (em). GFP, RFP, and phase-contrast images were taken from multiple spots on the glass slide. At least $1000 \mathrm{~B}$. subtilis and $E$. coli cells from multiple images were counted by ilastik (53) to quantify the degree of spatial proximity within regions of interest $(\mathrm{ROI})$.

\section{Statistical analysis}

We used an unpaired $t$-test to determine if there is a significant difference between the means of two groups in Figure 2J, 3C, and fig. S3C. We used a Mann-Whitney U Test to determine if two samples are derived from the same population in fig. S2D. Statistically different groups are denoted by ${ }^{*} p$-value $<0.05$, ${ }^{* *}$ if $p$-value $<0.01$, or ${ }^{* * *}$ if $p$-value $<0.001$. The Pearson correlation coefficient, $p$-value, and least-squares line were calculated to quantify the correlation between two variables in Figure $\mathbf{5}$ (A, C, E, and G), the inset in fig. S6, and fig. S8.

\section{ACKNOWLEDGEMENTS}

We would like to thank Jason Zuke and Zhengyi Chen for the assistance with the experiments, and Victor Zavala, Joshua Pulsipher, and Ryan Clark for helpful discussions about the 
computational model. This work was supported by the Defense Advanced Research Projects Agency (DARPA) Grant HR0011-19-2-0002.

\section{AUTHOR CONTRIBUTIONS}

Y.Y.C., B.M.B. and O.S.V. conceived the research. Y.Y.C. performed the experiments. O.S.V., B.M.B. and Y.Y.C. analyzed the data. O.S.V. and Y.Y.C. wrote the manuscript. Y.Y.C. and O.S.V. developed the model. J.M.P. constructed the Ec-C strain. O.S.V. secured the funding.

\section{COMPETING INTERESTS}

The authors declare no competing financial interests.

\section{REFERENCES}

1. S. M. Soucy, J. Huang, J. P. Gogarten, Horizontal gene transfer: Building the web of life. Nat. Rev. Genet. 16, 472-482 (2015).

2. J. Ropars, R. C. Rodríguez de la Vega, M. López-Villavicencio, J. Gouzy, E. Sallet, É. Dumas, S. Lacoste, R. Debuchy, J. Dupont, A. Branca, T. Giraud, Adaptive horizontal gene transfers between multiple cheese-associated fungi. Curr. Biol. 25, 2562-2569 (2015).

3. M. Clarke, L. Maddera, R. L. Harris, P. M. Silverman, F-pili dynamics by live-cell imaging. Proc. Natl. Acad. Sci. U. S. A. 105, 17978-17981 (2008).

4. S. Overballe-Petersen, K. Harms, L. A. A. Orlando, J. V. M. Mayar, S. Rasmussen, T. W. Dahl, M. T. Rosing, A. M. Poole, T. Sicheritz-Ponten, S. Brunak, S. Inselmann, J. de Vries, W. Wackernagel, O. G. Pybus, R. Nielsen, P. J. Johnsen, K. M. Nielsen, E. Willerslev, Bacterial natural transformation by highly fragmented and damaged DNA. Proc. Natl. Acad. Sci. U. S. A. 110, 19860-19865 (2013).

5. C. Johnston, B. Martin, G. Fichant, P. Polard, J.-P. Claverys, Bacterial transformation: distribution, shared mechanisms and divergent control. Nat. Rev. Microbiol. 12, 181-196 (2014).

6. N. A. Lerminiaux, A. D. S. Cameron, Horizontal transfer of antibiotic resistance genes in clinical environments. Can. J. Microbiol. 65, 34-44 (2019).

7. A. Jensen, O. Valdórsson, N. Frimodt-Møller, S. Hollingshead, M. Kilian, Commensal Streptococci serve as a reservoir for $\beta$-lactam resistance genes in Streptococcus pneumoniae. Antimicrob. Agents Chemother. 59, 3529-3540 (2015).

8. J. Sauerbier, P. Maurer, M. Rieger, R. Hakenbeck, Streptococcus pneumoniae R6 interspecies transformation: genetic analysis of penicillin resistance determinants and genome-wide recombination events. Mol. Microbiol. 86, 692-706 (2012).

9. R. Hakenbeck, T. Grebe, D. Zähner, J. B. Stock, $\beta$-lactam resistance in Streptococcus pneumoniae: penicillin-binding proteins and non-penicillin-binding proteins. Mol. Microbiol. 33, 673-678 (1999).

10. P. H. Brito, B. Chevreux, C. R. Serra, G. Schyns, A. O. Henriques, J. B. Pereira-Leal, Genetic competence drives genome diversity in Bacillus subtilis. Genome Biol. Evol. 10, 108-124 (2018).

11. R. M. Cooper, L. Tsimring, J. Hasty, Inter-species population dynamics enhance microbial horizontal gene transfer and spread of antibiotic resistance. Elife. 6, e25950 (2017).

12. S. Borgeaud, L. C. Metzger, T. Scrignari, M. Blokesch, The type VI secretion system of Vibrio cholerae fosters horizontal gene transfer. Science. 347, 63-67 (2015).

13. W.-Y. Wholey, T. J. Kochan, D. N. Storck, S. Dawid, Coordinated bacteriocin expression and competence in Streptococcus pneumoniae contributes to genetic adaptation through neighbor predation. PLoS Pathog. 12, e1005413 (2016).

14. X. Zhang, T. Jin, L. Deng, C. Wang, Y. Zhang, X. Chen, Stress-induced, highly efficient, 
donor cell-dependent cell-to-cell natural transformation in Bacillus subtilis. J. Bacteriol. 200, e00267-18 (2018).

15. G. D. Tribble, T. W. Rigney, D. H. V. Dao, C. T. Wong, J. E. Kerr, B. E. Taylor, S. Pacha, H. B. Kaplan, Natural competence is a major mechanism for horizontal DNA transfer in the oral pathogen Porphyromonas gingivalis. MBio. 3, e00231-11 (2012).

16. G. J. Stewart, C. A. Carlson, J. L. Ingraham, Evidence for an active role of donor cells in natural transformation of Pseudomonas stutzeri. J. Bacteriol. 156, 30-35 (1983).

17. J. H. Paul, J. M. Thurmond, M. E. Frischer, J. P. Cannon, Intergeneric natural plasmid transformation between E. coli and a marine Vibrio species. Mol. Ecol. 1, 37-46 (1992).

18. R. Frame, J. O. Bishop, The number of sex-factors per chromosome in Escherichia coli. Biochem. J. 121, 93-103 (1971).

19. C. L. Lee, D. S. W. Ow, S. K. W. Oh, Quantitative real-time polymerase chain reaction for determination of plasmid copy number in bacteria. J. Microbiol. Methods. 65, 258-267 (2006).

20. D. Mao, Y. Luo, J. Mathieu, Q. Wang, L. Feng, Q. Mu, C. Feng, P. J. J. Alvarez, Persistence of extracellular DNA in river sediment facilitates antibiotic resistance gene propagation. Environ. Sci. Technol. 48, 71-78 (2014).

21. K. Matsui, N. Ishii, Z. Kawabata, Release of extracellular transformable plasmid DNA from Escherichia coli cocultivated with algae. Appl. Environ. Microbiol. 69, 2399-2404 (2003).

22. M. G. Lorenz, D. Gerjets, W. Wackernagel, Release of transforming plasmid and chromosomal DNA from two cultured soil bacteria. Arch. Microbiol. 156, 319-326 (1991).

23. A. L. Ibáñez de Aldecoa, O. Zafra, J. E. González-Pastor, Mechanisms and regulation of extracellular DNA release and its biological roles in microbial communities. Front. Microbiol. 8, 1390 (2017).

24. J. C. Diaz Ricci, M. E. Hernández, Plasmid effects on Escherichia coli metabolism. Crit. Rev. Biotechnol. 20, 79-108 (2000).

25. H. Ingmer, C. Miller, S. N. Cohen, The RepA protein of plasmid pSC101 controls Escherichia coli cell division through the SOS response. Mol. Microbiol. 42, 519-526 (2001).

26. M. E. Patient, D. K. Summers, ColE1 multimer formation triggers inhibition of Escherichia coli cell division. Mol. Microbiol. 9, 1089-1095 (1993).

27. X.-Z. Zhang, Y.-H. P. Zhang, Simple, fast and high-efficiency transformation system for directed evolution of cellulase in Bacillus subtilis. Microb. Biotechnol. 4, 98-105 (2011).

28. N. Mirouze, Y. Desai, A. Raj, D. Dubnau, Spo0A P Imposes a Temporal Gate for the Bimodal Expression of Competence in Bacillus subtilis. PLoS Genet. 8, e1002586 (2012).

29. O. S. Venturelli, A. V Carr, G. Fisher, R. H. Hsu, R. Lau, B. P. Bowen, S. Hromada, T. Northen, A. P. Arkin, Deciphering microbial interactions in synthetic human gut microbiome communities. Mol. Syst. Biol. 14, e8157 (2018).

30. R. T. Jeters, G.-R. Wang, K. Moon, N. B. Shoemaker, A. A. Salyers, Tetracyclineassociated transcriptional regulation of transfer genes of the Bacteroides conjugative transposon CTnDOT. J. Bacteriol. 191, 6374-6382 (2009).

31. M. Prudhomme, L. Attaiech, G. Sanchez, B. Martin, J.-P. Claverys, Antibiotic stress induces genetic transformability in the human pathogen Streptoccus pneumoniae. Science. 313, 89-92 (2006).

32. X. Charpentier, E. Kay, D. Schneider, H. A. Shuman, Antibiotics and UV radiation induce competence for natural transformation in Legionella pneumophila. J. Bacteriol. 193, 1114-1121 (2011).

33. A. Lim, E. Lee, E. Yip, H. Lam, K. Hui, Effects of the stringent response on pBR322 plasmid copy number in Escherichia coli strains. J. Exp. Microbiol. Immunol. 9, 86-91 (2006). 
34. M. Au, J. Ip, I. Tao, M. A. Zhang, Evaluation of stringency elicited by antibiotics on plasmid copy number of pBR322 per genome in DH5a Escherichia coli cells. J. Exp. Microbiol. Immunol. 13, 83-88 (2009).

35. R. Rahmer, K. M. Heravi, J. Altenbuchner, Construction of a super-competent Bacillus

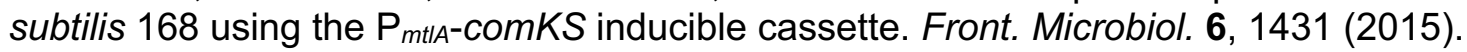

36. P. Tortosa, L. Logsdon, B. Kraigher, Y. Itoh, I. Mandic-Mulec, D. Dubnau, Specificity and genetic polymorphism of the Bacillus competence quorum-sensing system. J. Bacteriol. 183, 451-460 (2001).

37. J.-P. Claverys, M. Prudhomme, B. Martin, Induction of Competence Regulons as a General Response to Stress in Gram-Positive Bacteria. Annu. Rev. Microbiol. 60, 451475 (2006).

38. R. López-igual, J. Bernal-bayard, A. Rodríguez-patón, J. Ghigo, D. Mazel, Engineered toxin-intein antimicrobials can selectively target and kill antibiotic-resistant bacteria in mixed populations. Nat. Biotechnol. 37, 755-760 (2019).

39. D. Bikard, C. W. Euler, W. Jiang, P. M. Nussenzweig, G. W. Goldberg, X. Duportet, V. A. Fischetti, L. A. Marraffini, Exploiting CRISPR-Cas nucleases to produce sequencespecific antimicrobials. Nat. Biotechnol. 32, 1146-1150 (2014).

40. J. Slager, M. Kjos, L. Attaiech, J.-W. Veening, Antibiotic-induced replication stress triggers bacterial competence by increasing gene dosage near the origin. Cell. 157, 395406 (2014).

41. A. Domenech, A. R. Brochado, V. Sender, K. Hentrich, B. Henriques-Normark, A. Typas, J.-W. Veening, Proton motive force disruptors block bacterial competence and horizontal gene transfer. Cell Host Microbe. 27, 544-555 (2020).

42. A. K. Møller, M. P. Leatham, T. Conway, P. J. M. Nuijten, L. A. M. de Haan, K. A. Krogfelt, P. S. Cohen, An Escherichia coli MG1655 lipopolysaccharide deep-rough core mutant grows and survives in mouse cecal mucus but fails to colonize the mouse large intestine. Infect. Immun. 71, 2142-2152 (2003).

43. G. P. Dubey, S. Ben-Yehuda, Intercellular nanotubes mediate bacterial communication. Cell. 144, 590-600 (2011).

44. K. Jann, G. Schmidt, E. Blumenstock, K. Vosbeck, Escherichia coli adhesion to Saccharomyces cerevisiae and mammalian cells: role of piliation and surface hydrophobicity. Infect. Immun. 32, 484-489 (1981).

45. Y. Suzuki, S. Nishijima, Y. Furuta, J. Yoshimura, W. Suda, K. Oshima, M. Hattori, S. Morishita, Long-read metagenomic exploration of extrachromosomal mobile genetic elements in the human gut. Microbiome. 7, 119 (2019).

46. T. Blazejewski, H.-I. Ho, H. H. Wang, Synthetic sequence entanglement augments stability and containment of genetic information in cells. Science. 365, 595-598 (2019).

47. E. Yaffe, D. A. Relman, Tracking microbial evolution in the human gut using Hi-C reveals extensive horizontal gene transfer, persistence and adaptation. Nat. Microbiol. 5, 343353 (2020).

48. R. G. Egbert, H. S. Rishi, B. A. Adler, D. M. McCormick, E. Toro, R. T. Gill, A. P. Arkin, A versatile platform strain for high-fidelity multiplex genome editing. Nucleic Acids Res. 47, 3244-3256 (2019).

49. R. Lutz, H. Bujard, Independent and tight regulation of transcriptional units in Escherichia coli via the LacR/O, the TetR/O and AraC/I1-I2 regulatory elements. Nucleic Acids Res. 25, 1203-1210 (1997).

50. T. S. Lee, R. A. Krupa, F. Zhang, M. Hajimorad, W. J. Holtz, N. Prasad, S. K. Lee, J. D. Keasling, BglBrick vectors and datasheets: A synthetic biology platform for gene expression. J. Biol. Eng. 5, 12 (2011).

51. W. Overkamp, K. Beilharz, R. D. O. Weme, A. Solopova, H. Karsens, Á. T. Kovács, J. Kok, O. P. Kuipers, J.-W. Veening, Benchmarking various green fluorescent protein 
variants in Bacillus subtilis, Streptococcus pneumoniae, and Lactococcus lactis for live cell imaging. Appl. Environ. Microbiol. 79, 6481-6490 (2013).

52. B. J. Haijema, J. Hahn, J. Haynes, D. Dubnau, A ComGA-dependent checkpoint limits growth during the escape from competence. Mol. Microbiol. 40, 52-64 (2001).

53. S. Berg, D. Kutra, T. Kroeger, C. N. Straehle, B. X. Kausler, C. Haubold, M. Schiegg, J. Ales, T. Beier, M. Rudy, K. Eren, J. I. Cervantes, B. Xu, F. Beuttenmueller, A. Wolny, C. Zhang, U. Koethe, F. A. Hamprecht, A. Kreshuk, ilastik: interactive machine learning for (bio)image analysis. Nat. Methods. 16, 1226-1232 (2019).

54. O. Fridman, A. Goldberg, I. Ronin, N. Shoresh, N. Q. Balaban, Optimization of lag time underlies antibiotic tolerance in evolved bacterial populations. Nature. 513, 418-421 (2014).

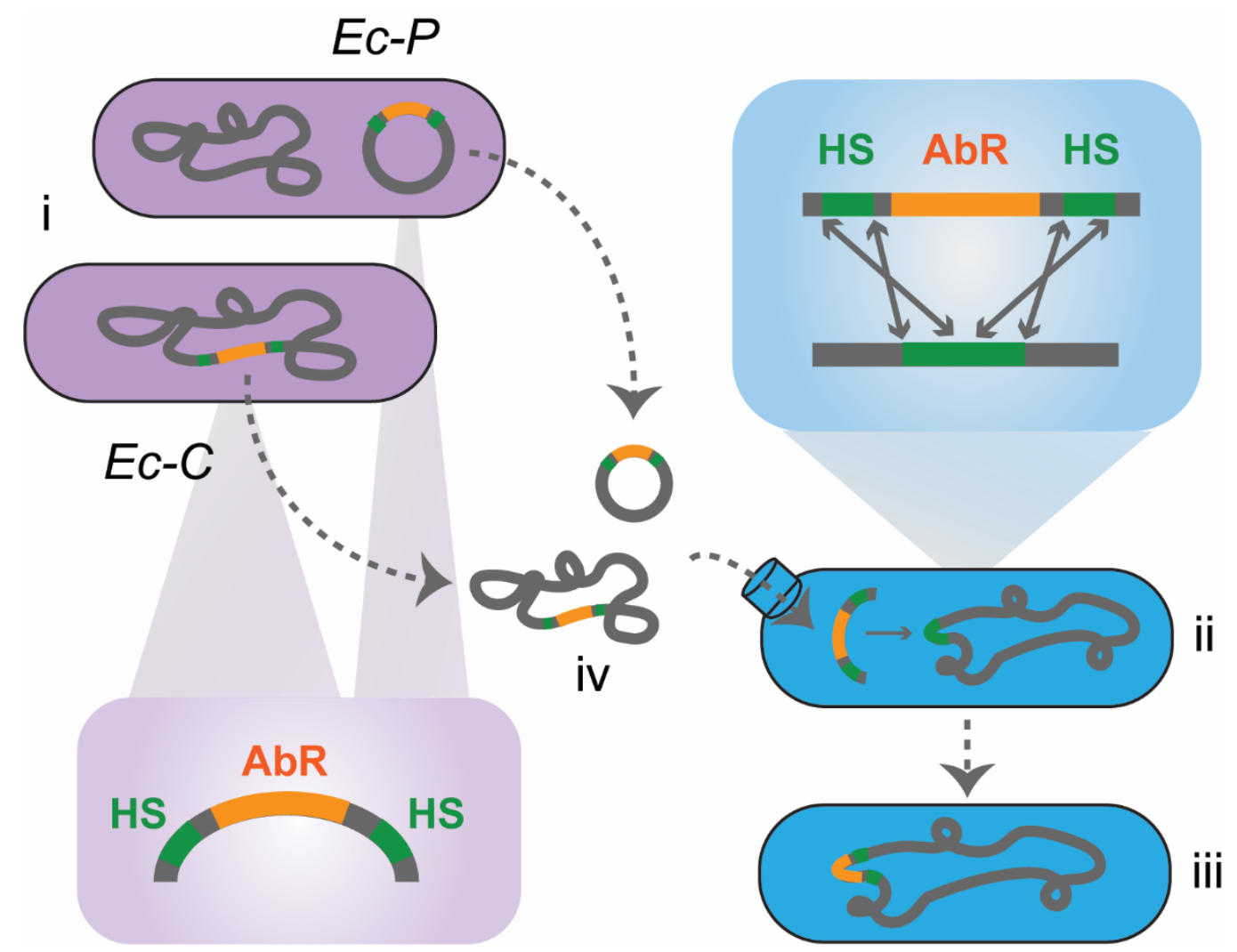

Figure 1. Horizontal gene transfer from $E$. coli to $B$. subtilis in the synthetic bacterial consortium. E. coli donor has an integration cassette that is composed of an antibiotic resistance gene (AbR) flanked by two homologous sequences (HS) of $B$. subtilis. The integration cassette is either on the plasmid $(E c-P)$ or genome $(E c-C)$. Competent $B$. subtilis can take up DNA and the $A b R$ can be integrated into $B$. subtilis genome via homologous recombination. To characterize the gene transfer process, E. coli (i), B. subtilis (ii), transformed B. subtilis (iii), and eDNA (iv) were measured over time. 
A

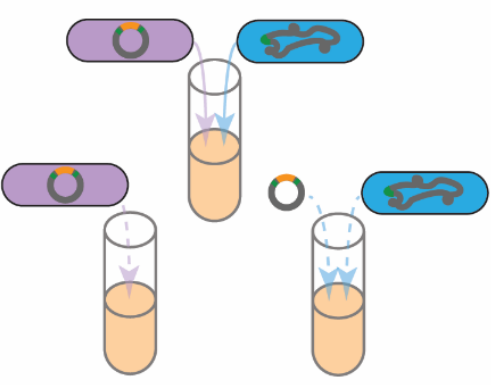

C

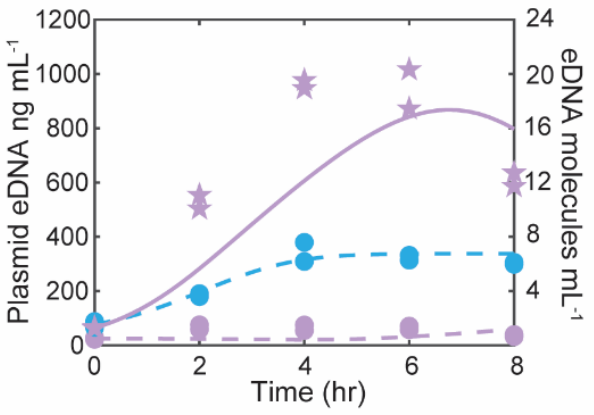

$E$

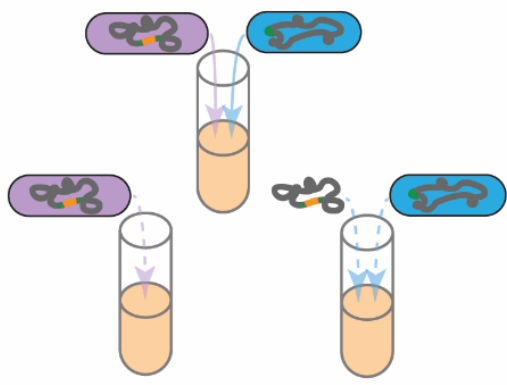

G

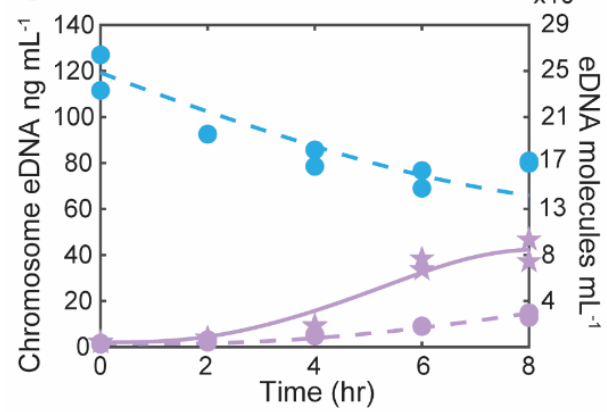

I

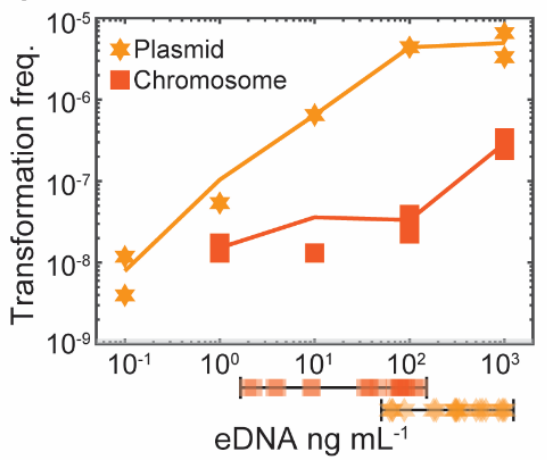

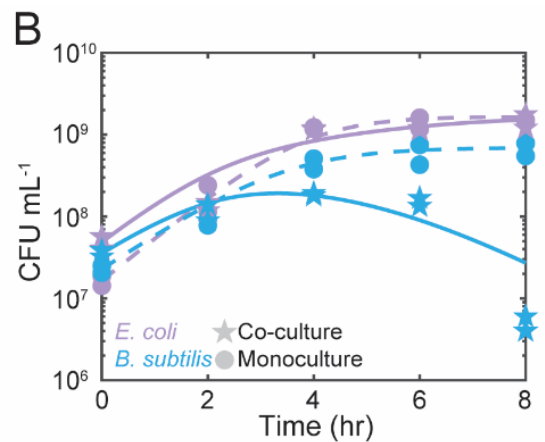

D

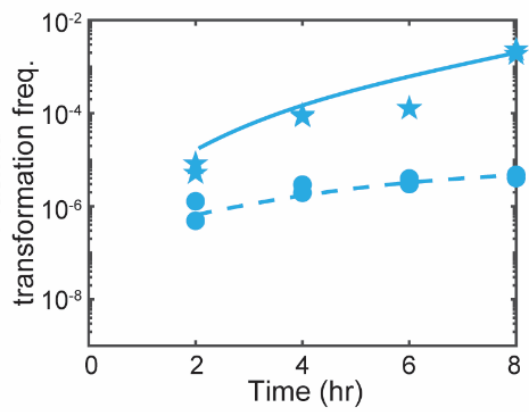

$\mathrm{F}$

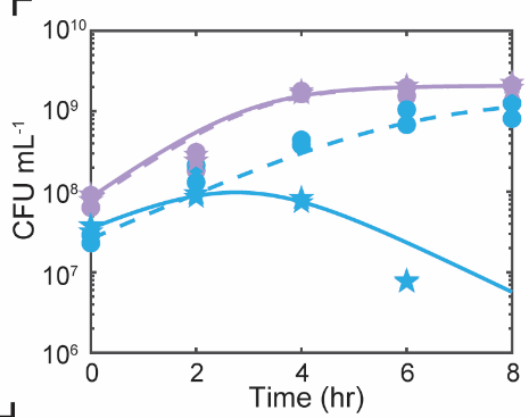

$\mathrm{H}$

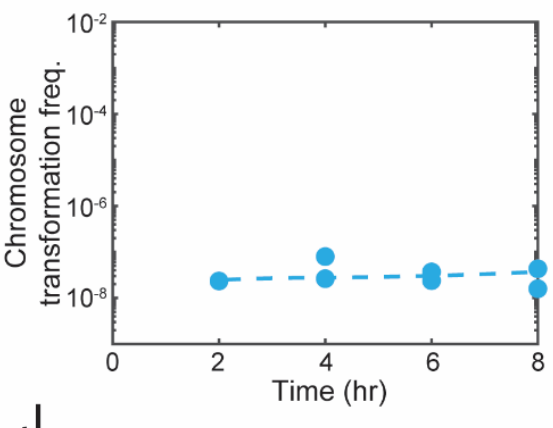

J

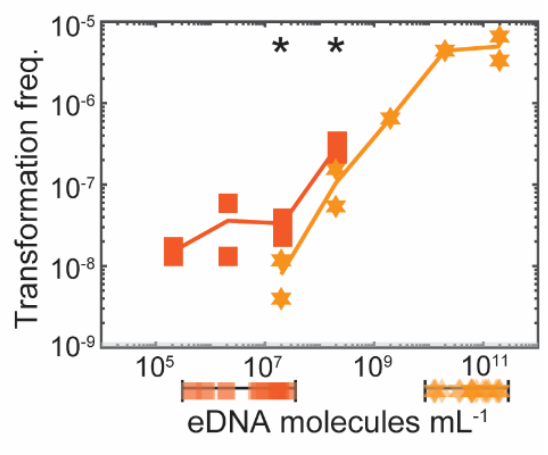


702

703

704

705

706

707
Figure 2. Temporal characterization of gene transfer in the synthetic microbial community. (A) Schematic of experimental design to characterize the temporal changes in plasmid gene transfer in the $B$. subtilis (blue) monoculture in the presence of purified plasmid DNA (100 ng mL' ${ }^{1}$ ) or co-culture of Ec-P (purple) and $B$. subtilis. Extracellular plasmid release was characterized in the $E c-P$ monoculture. (B) Time-series measurements of $E_{C}-P$ and $B$. subtilis CFU mL ${ }^{-1}$ in the monocultures (dashed) and co-culture (solid). Data points represent experimental measurements and lines denote model fits to the data. (C) Time-series measurements of extracellular plasmid DNA in the Ec-P and $B$. subtilis monocultures (dashed) or co-culture (solid). Data points represent experimental measurements and lines denote model fits to the data. (D) Time-series measurements of plasmid transformation frequency for the $B$. subtilis monoculture (dashed) in the presence of purified plasmid DNA $100 \mathrm{ng} \mathrm{mL}^{-1}$ ) or co-culture with $E c-P$ (solid). Data points represent experimental measurements and lines denote model fits to the data. (E) Schematic of experimental design to characterize the temporal changes in chromosomal gene transfer in the $B$. subtilis (blue) monoculture supplemented with purified genomic Ec-C DNA (100 ng mL $\left.{ }^{-1}\right)$ or coculture of $B$. subtilis and $E c-C$ (purple). (F) Time-series measurements of $E c-C$ and $B$. subtilis CFU mL $\mathrm{m}^{-1}$ in monoculture (dashed) and co-culture (solid). Data points represent experimental measurements and lines denote model fits to the data. (G) Time-series measurements of extracellular chromosomal DNA in the $E c-C$ and $B$. subtilis monocultures (dashed) and co-culture (solid). Data points and lines denote experimental measurements and model fits, respectively. (H) Time-series measurements of chromosome transformation frequency in the $B$. subtilis monoculture supplemented with purified genomic Ec-C DNA $\left(100 \mathrm{ng} \mathrm{mL}^{-1}\right)$ or the co-culture (no transformants observed). Data points and lines represent experimental measurements and model fits to the data, respectively. (I) Relationship between purified plasmid or chromosome concentration and transformation frequency in the $B$. subtilis monoculture at $6 \mathrm{hr}$. Horizontal lines indicate the range of extracellular plasmid and chromosome concentrations measured in the $B$. subtilis monoculture with purified DNA (100 $\mathrm{ng} \mathrm{mL}^{-1}$ ) and co-cultures with Ec-P or Ec-C (Fig. 2, C and $\mathbf{G})$. Data points and lines denote experimental measurements experimental measurements and the average of two biological replicates. $(\mathbf{J})$ Relationship between eDNA copy number per $\mathrm{mL}$ of purified plasmid or chromosome and transformation frequency at $6 \mathrm{hr}$ in the $B$. subtilis monoculture. All conditions were supplemented with $50 \mathrm{mM}$ xylose. 
A

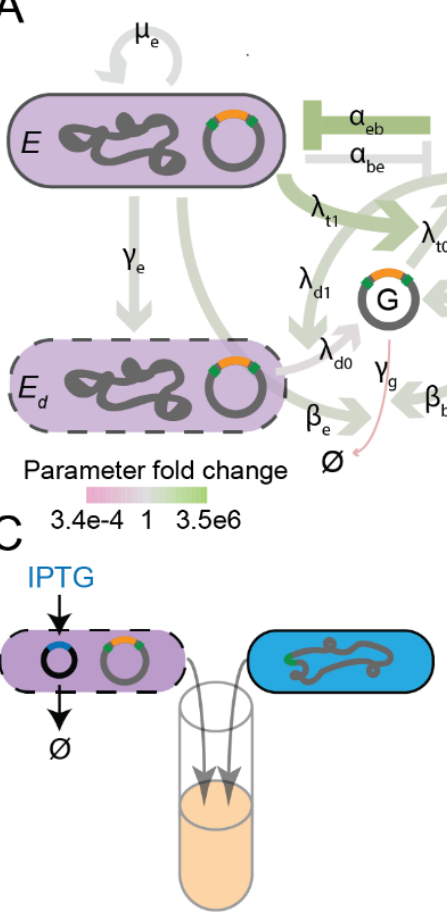

$E$

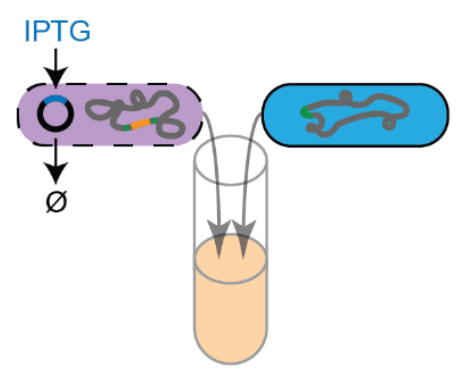

G

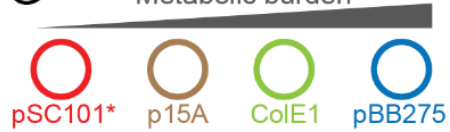

SC101* p15A ColE1 pBB275

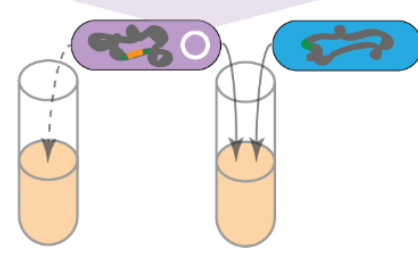

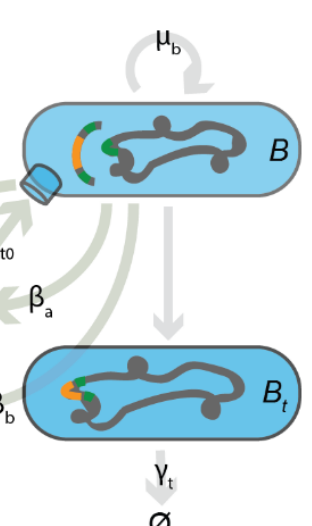

$\varnothing$

B
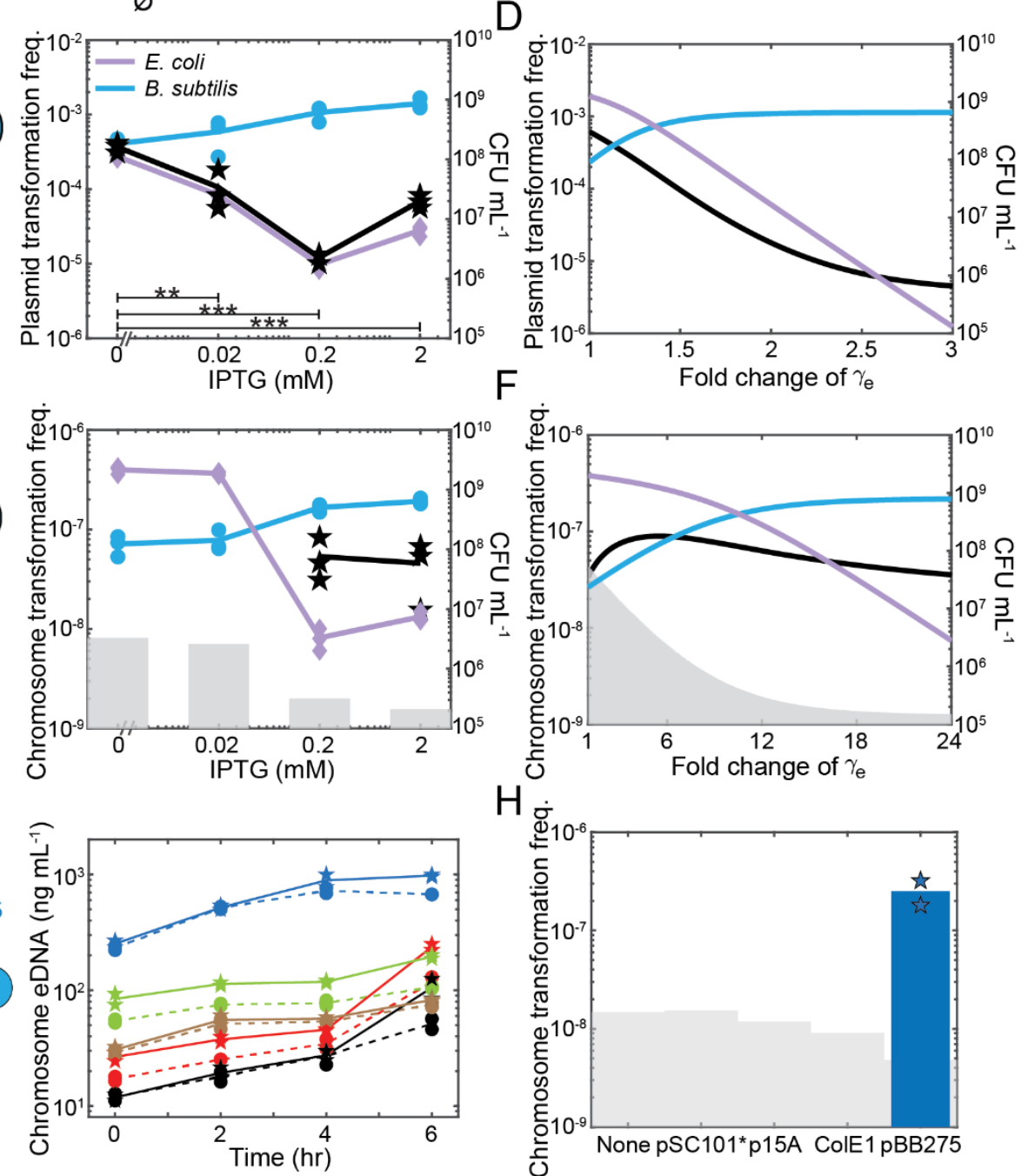

$\mathrm{H}$

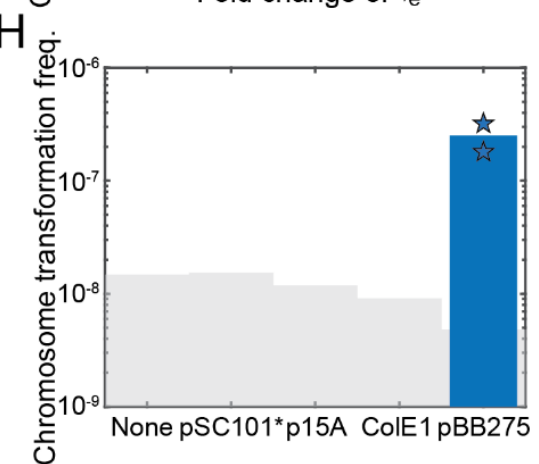

Figure 3. Model prediction on the role of donor in plasmid-mediated and chromosomemediated gene transfer. (A) Schematic of the key interactions in the dynamic computational model of growth and horizontal gene transfer. The model species include $E$. coli $(E)$, dead $E$. coli $\left(E_{d}\right)$, B. subtilis $(B)$, eDNA $(G)$, and transformed $B$. subtilis $\left(B_{t}\right)$. The line width and color of each edge are proportional to the fold change of each parameter in the plasmid to chromosome model. 
frequency in the plasmid or chromosome model at $6 \mathrm{hr}$ for $20 \%$ increase or decrease in the parameter value compared to the estimated parameter set. (C) Schematic of a co-culture experiment of $E c-P$ harboring an IPTG-inducible lysis gene and $B$. subtilis (left). Relationship between IPTG concentration and plasmid transformation frequency or CFU mL ${ }^{-1}$ (right) at $6 \mathrm{hr}$ in the co-culture. Transformation frequency exhibited a decreasing trend with IPTG concentration ( $p$-value $\left.<0.01{ }^{* *}\right)$ or $p$-value $\left.<0.001{ }^{* * *}\right)$ using an unpaired $t$-test. (D) Ec- $P$ model prediction of the concentration (CFU mL ${ }^{-1}$ ) of $E$ and $B$, or plasmid transformation frequency at $6 \mathrm{hr}$ as a function of the fold change in the Ec-P death rate $\gamma_{e}$ compared to the estimated parameter value. (E) Schematic of a co-culture experiment of $E c-C$ harboring an IPTG-inducible lysis gene and $B$. subtilis (left). Relationship between IPTG concentration and chromosome transformation frequency or CFU mL $\mathrm{m}^{-1}$ (right) at $6 \mathrm{hr}$ in the co-culture. Transformants were detected at higher IPTG concentrations. Shaded regions denote the detection limits for transformation frequency. (F)

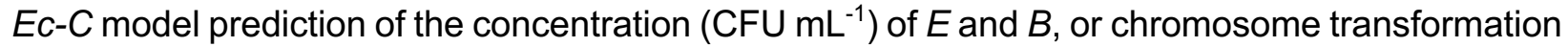
frequency at $6 \mathrm{hr}$ as a function of the fold change in the Ec-C death rate $\gamma_{e}$ compared to the estimated parameter value. We assume that the detection limit of transformants in the model is 1 CFU mL ${ }^{-1} B_{t}$ divided by the sum of $B_{t}$ and $B$ CFU mL ${ }^{-1}$. (G) Schematic of experimental design (left) of co-cultures composed of $E c-C$ harboring a single plasmid with variable origin of replication and $B$. subtilis (solid) or Ec-C monoculture (dashed). Time-series measurements of extracellular chromosome concentration in the $E c-C$ monocultures or co-cultures. (H) Chromosome transformation frequency at $4 \mathrm{hr}$ in each co-culture of $E c-C$ harboring different plasmids and $B$. subtilis. Shaded area indicates the transformation frequency detection limit. Lines and bars in Fig. supplemented with $50 \mathrm{mM}$ xylose. 

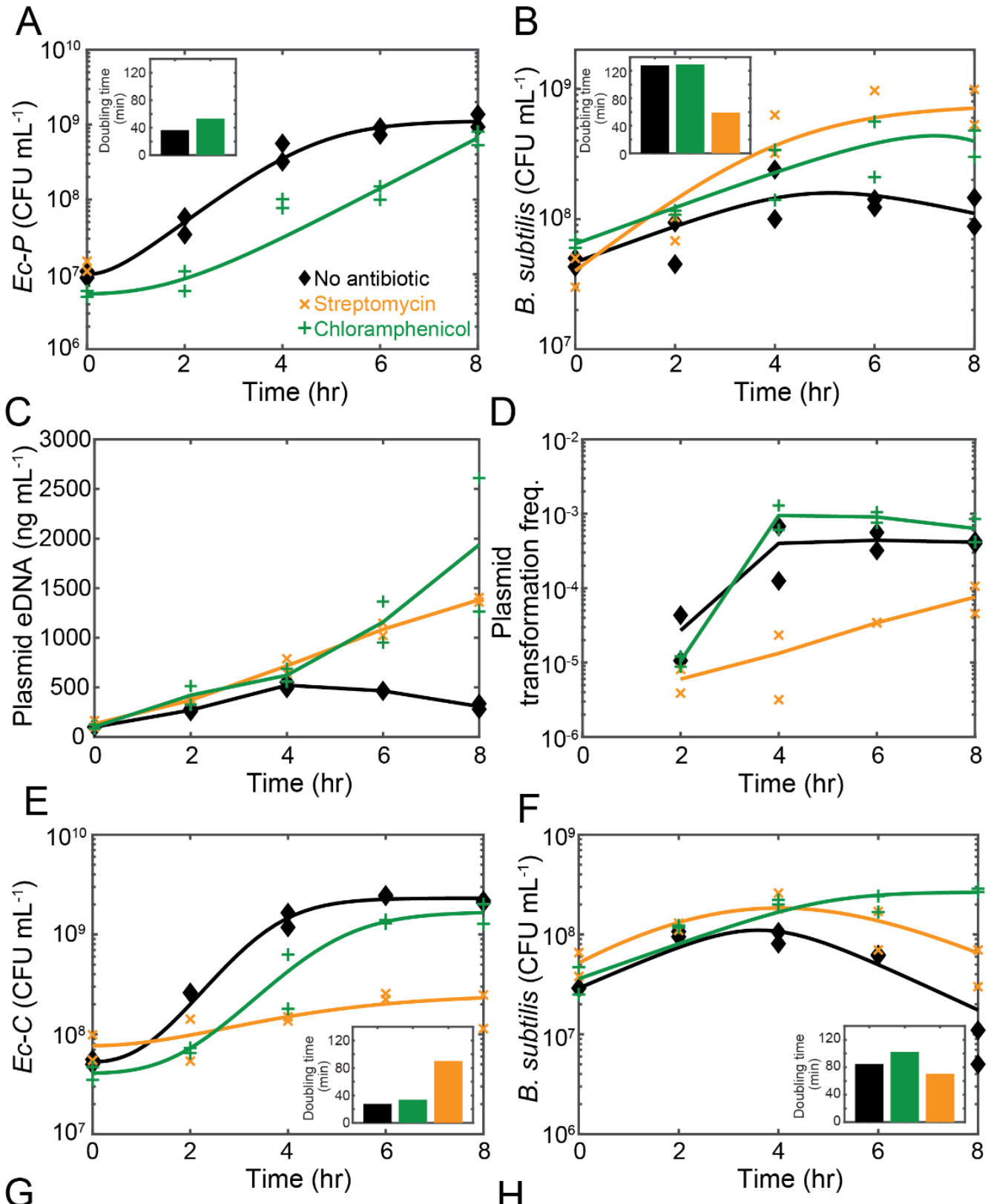

$\mathrm{F}$
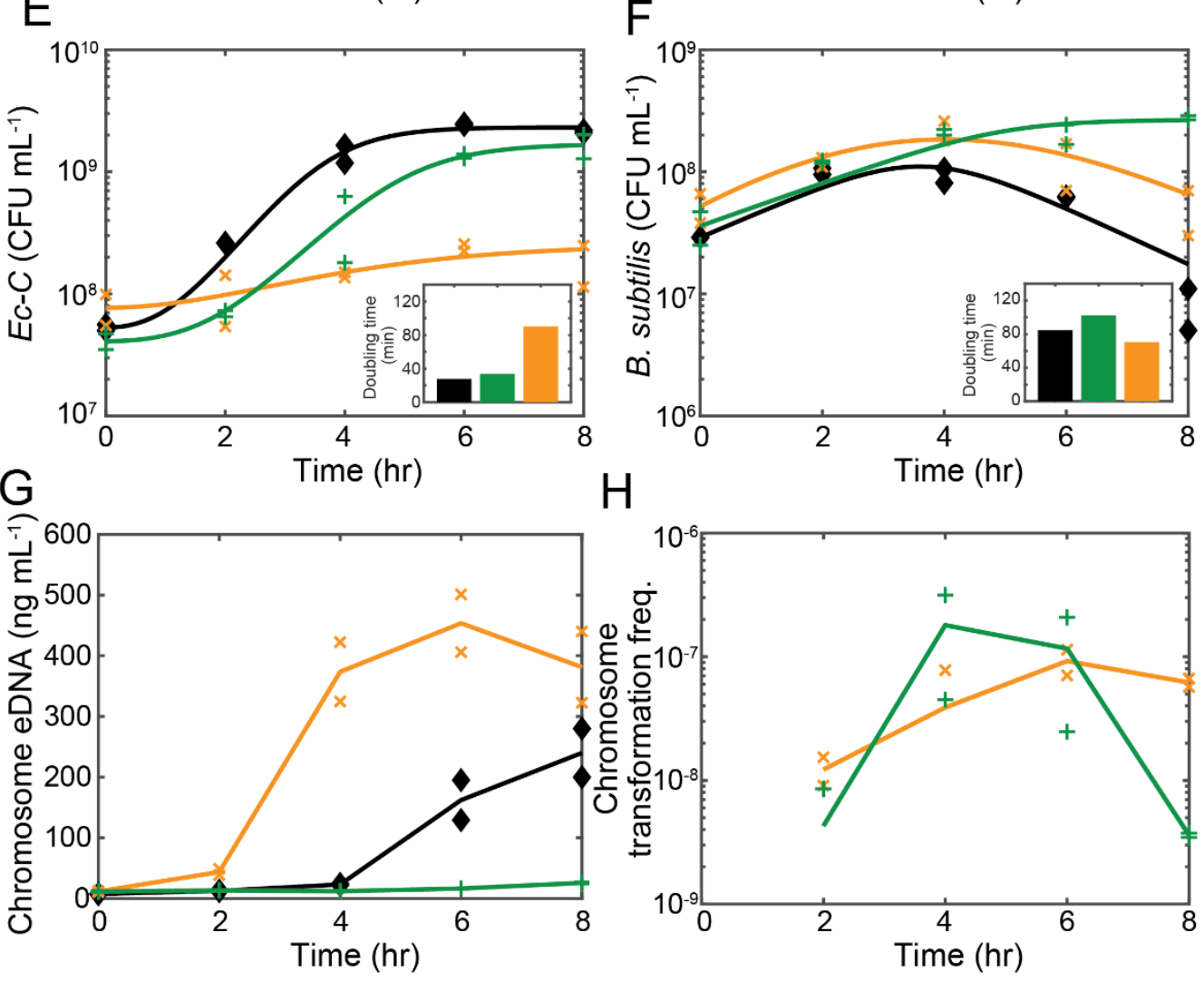
Figure 4. Impact of antibiotics on the dynamics of plasmid and chromosomal gene transfer in the synthetic microbial community. (A) Time-series measurements of $E c-P C F U \mathrm{~mL}^{-1}$ in coculture with $B$. subtilis in the presence of streptomycin $\left(10 \mu \mathrm{g} \mathrm{mL}^{-1}\right)$, chloramphenicol $\left(5 \mu \mathrm{g} \mathrm{mL}^{-1}\right)$ or no antibiotic. Data points and lines represent experimental measurements and growth model fits to the data. Inset: Inferred doubling times based on the growth models. (B) Time-series measurements of $B$. subtilis CFU mL ${ }^{-1}$ in the co-culture with $E c-P$ in the presence of streptomycin $\left(10 \mu \mathrm{g} \mathrm{mL} \mathrm{m}^{-1}\right)$, chloramphenicol $\left(5 \mu \mathrm{g} \mathrm{mL}^{-1}\right)$ or no antibiotic. Data points and lines represent experimental measurements and growth model fits to the data. Inset: Inferred doubling time based on the growth models. (C) Time-series measurements of extracellular plasmid DNA in the Ec-P and $B$. subtilis co-culture. Data points and lines represent experimental measurements and averages of biological replicates, respectively. (D) Time-series measurements of plasmid transformation frequency in the Ec-P and $B$. subtilis co-cultures. Data points and lines denote experimental measurements and mean of biological replicates, respectively. (E) Time-series measurements of $E c-C$ CFU mL ${ }^{-1}$ in co-culture with $B$. subtilis in the presence of streptomycin (10 $\left.\mu \mathrm{g} \mathrm{mL} \mathrm{m}^{-1}\right)$, chloramphenicol $\left(5 \mu \mathrm{g} \mathrm{mL}^{-1}\right)$ or no antibiotic. Data points and lines represent experimental measurements and growth model fits to the data. Inset: Inferred doubling times based on the growth models. (F) Time-series measurements of $B$. subtilis CFU $\mathrm{mL}^{-1}$ in the coculture with $E c-C$ in the presence of streptomycin $\left(10 \mu \mathrm{g} \mathrm{mL}^{-1}\right)$, chloramphenicol $\left(5 \mu \mathrm{g} \mathrm{mL}^{-1}\right)$ or no antibiotic. Data points and lines represent experimental measurements and growth model fits to the data. Inset: Inferred doubling time based on the growth models. (G) Time-series measurements of extracellular chromosome DNA in the co-culture with $E c-C$. Data points and lines denote experimental measurements and mean of biological replicates, respectively. $(\mathbf{H})$ Time-series measurements of chromosome transformation frequency in the $E c-C$ and $B$. subtilis co-culture. Data points and lines denote experimental measurements and mean of biological replicates, respectively. All conditions were supplemented with $50 \mathrm{mM}$ xylose. 
A

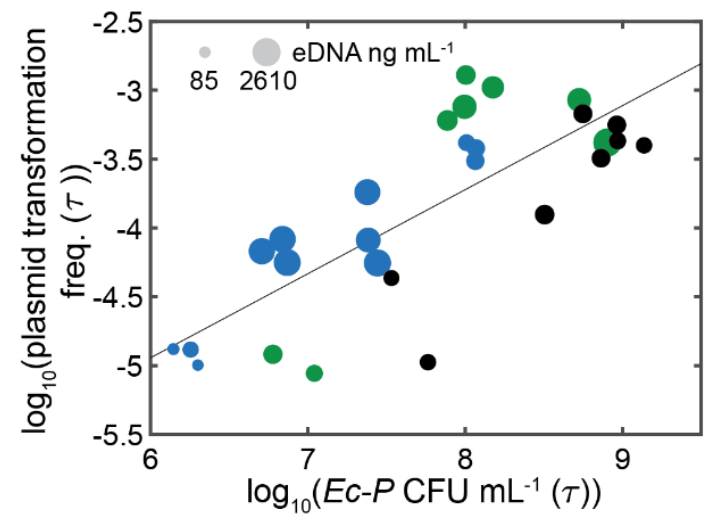

C

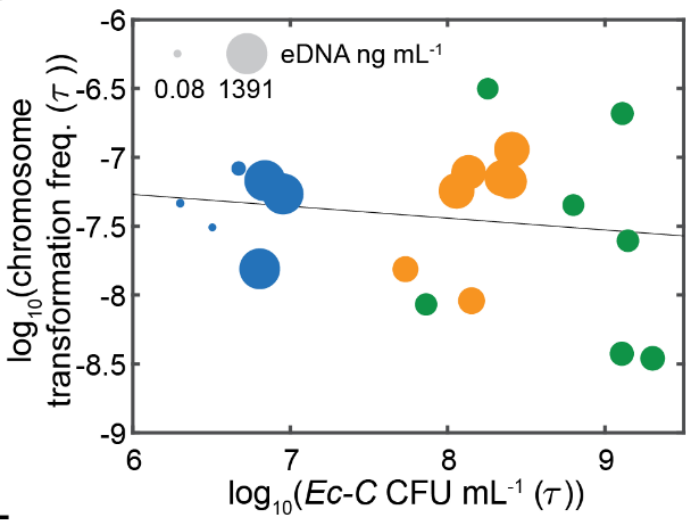

E

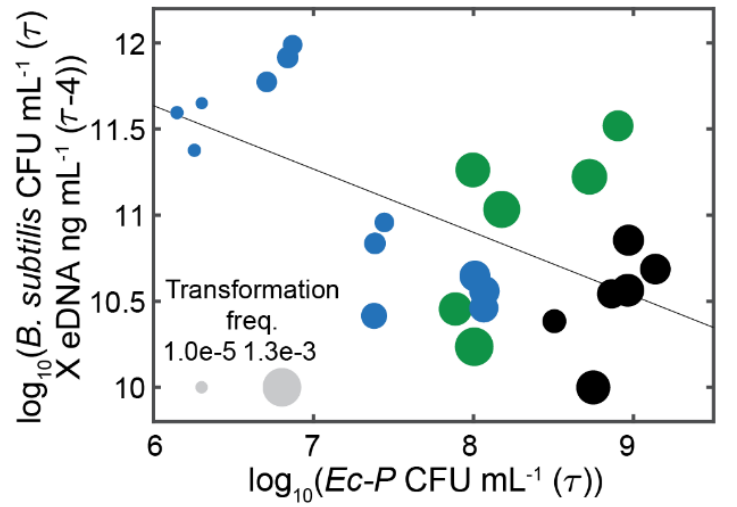

G

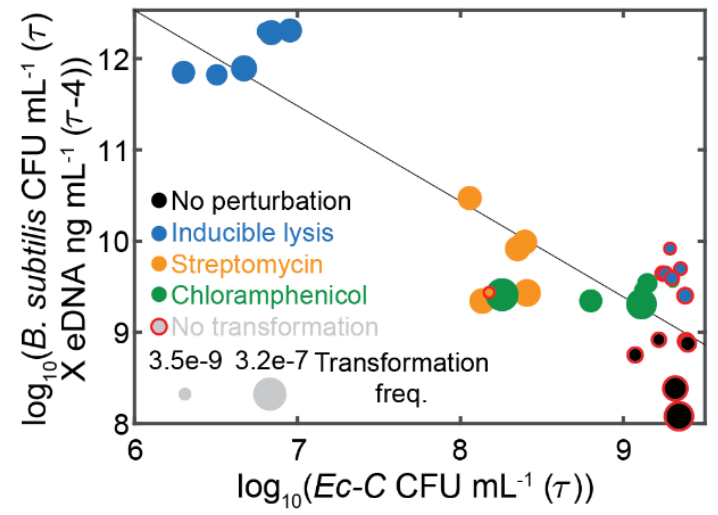

$\mathrm{B}$

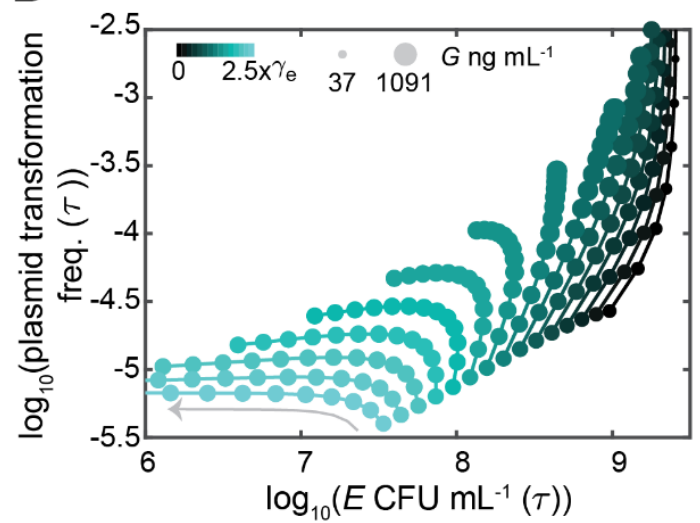

D

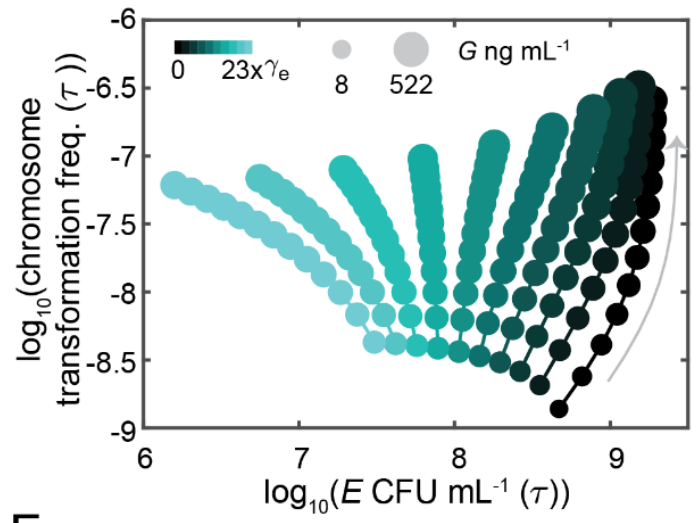

F

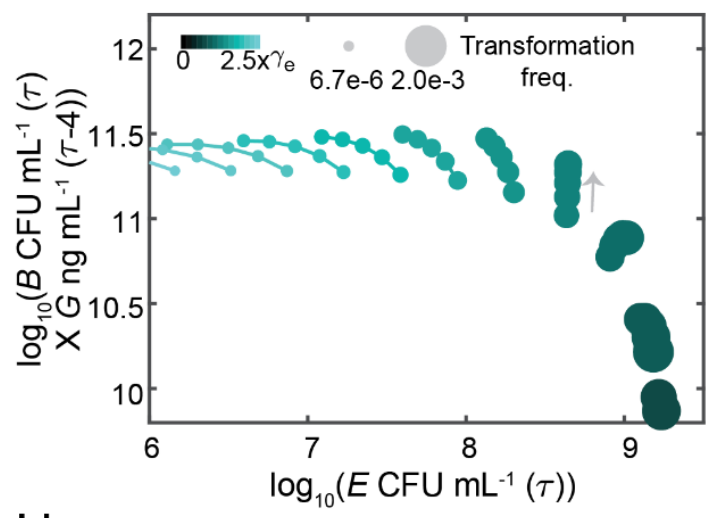

H

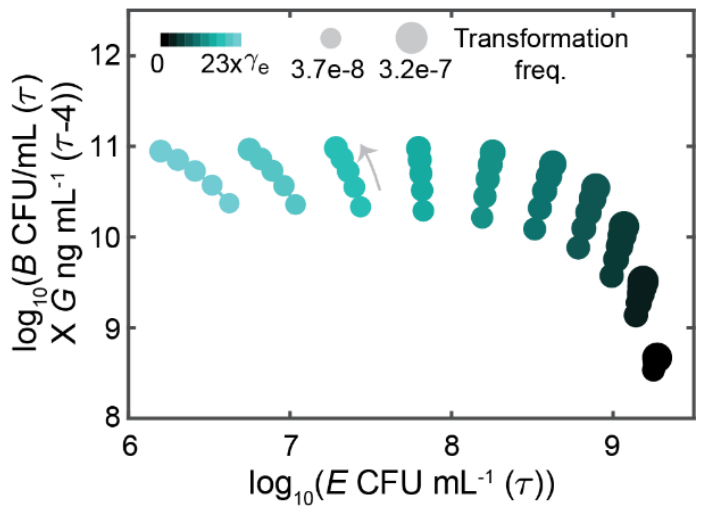


Figure 5. Global trends in transformation frequency, E. coli CFU, B. subtilis CFU, and eDNA concentration across different experimental datasets. (A) Scatter plot of $E c-P$ CFU mL ${ }^{-1}$ and plasmid transformation frequency (Pearson correlation coefficient $r=0.78, p$-value $=9.37 \mathrm{e}-7$ ). The size of each data point is proportional to the eDNA concentration. (B) Scatter plot of plasmid model prediction of the relationship between $E$ concentration and transformation frequency for a broad range of $E$ death rates $\gamma_{e}$. Data points connected by separate lines denote different values of $\gamma_{e}$. Gray arrow indicates the direction of time. (C) Scatter plot of Ec-C CFU mL $\mathrm{mL}^{-1}$ and chromosome transformation frequency. The size of the data point is proportional to the eDNA concentration. (D) Scatter plot of chromosome model prediction of the relationship between $E$ concentration and transformation frequency for a broad range of $E$ death rates $\gamma_{e}$. Data points connected by separate lines denote different values of $\gamma_{e}$. Gray arrow indicates the direction of time. (E) Scatter plot of Ec-P CFU mL ${ }^{-1}$ and the product of $B$. subtilis CFU mL $\mathrm{m}^{-1}$ and time-lagged eDNA concentration (Pearson correlation coefficient $r=-0.61, p$-value $=0.00163$ ). The size of each datapoint is proportional the transformation frequency. $(\mathbf{F})$ Scatter plot of the plasmid model prediction of the relationship between $E$ and the product of $B$ and time-lagged $G$ concentration across a broad range of $E$ death rates $\gamma_{e}$. Data points connected by separate lines denote different values of $\gamma_{e}$. The size of each data point is proportional to the transformation frequency. Gray arrow indicates the direction of time. (G) Scatter plot of EC-C CFU mL $\mathrm{m}^{-1}$ and the product of $B$. subtilis CFU mL $\mathrm{mL}^{-1}$ and time-lagged extracellular chromosome concentration (Pearson correlation coefficient $r=-0.89, p$-value $=2.94 \mathrm{e}-11$ ). The size of each data point is proportional to the transformation frequency. Data points with red outlines denote measurements where no $B$. subtilis transformants were detected and the size of these data points are proportional to the detection limit. $(\mathbf{H})$ Scatter plot of the chromosome model prediction of the relationship between $E$ and the product of $B$ and time-lagged $G$ concentration across a broad range of $E$ death rates $\gamma_{e}$. Data points connected by separate lines denote different values of $\gamma_{e}$. The size of each data point is proportional to the transformation frequency. The gray arrow indicates the direction of time. All conditions were supplemented with $50 \mathrm{mM}$ xylose. 